\title{
CENTRAL-UPWIND SCHEMES FOR THE SAINT-VENANT SYSTEM
}

\author{
Alexander Kurganov ${ }^{1}$ And Doron Levy ${ }^{2}$
}

\begin{abstract}
We present one- and two-dimensional central-upwind schemes for approximating solutions of the Saint-Venant system with source terms due to bottom topography. The Saint-Venant system has steady-state solutions in which nonzero flux gradients are exactly balanced by the source terms. It is a challenging problem to preserve this delicate balance with numerical schemes. Small perturbations of these states are also very difficult to compute. Our approach is based on extending semi-discrete central schemes for systems of hyperbolic conservation laws to balance laws. Special attention is paid to the discretization of the source term such as to preserve stationary steady-state solutions. We also prove that the second-order version of our schemes preserves the nonnegativity of the height of the water. This important feature allows one to compute solutions for problems that include dry areas.
\end{abstract}

Mathematics Subject Classification. 65M06, 35L65.

Received: June 20, 2001. Revised: February 17, 2002.

\section{INTRODUCTION}

The Saint-Venant system, which was introduced in [36], is commonly used to model flows in rivers or coastal areas. This system describes the flow as a conservation law with an additional source term. In one space dimension it takes the form

$$
\left\{\begin{array}{l}
h_{t}+(h u)_{x}=0 \\
(h u)_{t}+\left(h u^{2}+\frac{1}{2} g h^{2}\right)_{x}=-g h B_{x}
\end{array}\right.
$$

where $B(x)$ represents the bottom elevation, $h$ is the fluid depth above the bottom, $u$ denotes the velocity, and $g$ is the gravitational constant. More complete systems were recently derived from the Navier-Stokes equations [12].

Similarly to other balance laws, the Saint-Venant system admits steady-state solutions in which nonzero flux gradients are exactly balanced by the source terms. Such steady-states as well as their perturbations, are difficult to capture numerically. Standard numerical schemes for conservation laws will, in general, fail to preserve the delicate balance between the fluxes and the source terms.

\footnotetext{
Keywords and phrases. Saint-Venant system, shallow water equations, high-order central-upwind schemes, balance laws, conservation laws, source terms.

1 Department of Mathematics, University of Michigan, Ann Arbor, MI 48109-1109 and Mathematics Department, Tulane University, New Orleans, LA 70118, USA. e-mail: kurganov@math.tulane.edu

2 Department of Mathematics, Stanford University, Stanford, CA 94305-2125, USA. e-mail: dlevy@math.stanford.edu
} 
Recently, LeVeque et al. $[23,24]$ proposed a method which preserves stationary steady-states. In this method, a Riemann problem is introduced in the center of each grid cell such that the flux difference exactly cancels the source term. In order to compute the new half-cell averaged values, one has to solve a nonlinear algebraic problem. It was shown by LeVeque in [23] that there exist cases where his iterative method does not converge. A different approach, but one that is still based on rather complicated Riemann problem solvers, was taken by Gallouët et al. in [11]. Kinetic schemes were proved to keep a nonnegative water height, satisfy an entropy inequality and to preserve stationary steady-states. First-order kinetic solvers were proposed by Perthame et al. in $[5,34]$. High-order kinetic schemes may satisfy only part of these properties and are of a relatively complicated form. For other related works on balance laws we refer to $[6,13,16,35]$.

In this paper we present a new, high-order central-upwind scheme for the Saint-Venant system. Our method is based on the schemes developed in [18] for approximating solutions of multidimensional systems of conservation laws. Careful attention is being paid to the discretization of the source term in order to imitate the differential properties on the numerical level and preserve stationary steady-state solutions. We also prove that the computed water height remains nonnegative with the proposed second-order scheme. This is especially important when the water flows out of a certain domain, and dry states $(h \sim 0)$ appear.

Central schemes for conservation laws have recently attracted a lot of attention due to their simplicity, efficiency and robustness. These Godunov-type schemes are based on an exact evolution of an approximate piecewise polynomial reconstruction, do not require any (approximate) Riemann problem solvers, and can be therefore used as black-box solvers for a variety of problems. The prototype central scheme is the first-order Lax-Friedrichs scheme [10]. Its staggered, second-order, one-dimensional (1D) generalization was proposed by Nessyahu and Tadmor in [32]. Extensions to two space dimensions were obtained in $[3,15]$. High-order, unstructured grids and multidimensional versions can be found in $[4,7,25,26,30]$. Staggered central schemes for the 1D Saint-Venant system were proposed in [27]. Note that these methods do not preserve stationary steady-states.

The major drawback of staggered central schemes is the relatively large numerical dissipation $\left(\sim \mathcal{O}\left(\frac{(\Delta x)^{2 r}}{\Delta t}\right)\right.$, where $r$ is a formal order of accuracy), which significantly increases if the time step, $\Delta t$, is forced to be much smaller than the spatial scale $\Delta x$. The same problem occurs if the solution is computed for large times as happens, for example, in steady-state computations. Kurganov and Tadmor [21] introduced a new class of semi-discrete central schemes, which enjoy a much smaller numerical dissipation $\left(\sim \mathcal{O}\left((\Delta t)^{2 r-1}\right)\right)$. This is achieved by utilizing local speeds of propagation for more precise estimate of the width of the Riemann fans, but still without any attempt to (approximately) solve Riemann problems. The third-order extension of these schemes was proposed in [17], and their genuinely multidimensional generalization was developed in [19]. The numerical dissipation can be further reduced by considering the one-sided local speeds. This results in the central-upwind schemes $[18,20]$.

The paper is organized as follows. In Section 2 we outline the model equations that will be used throughout the paper. We proceed in Section 3, where we present the numerical methods for balance laws. In Section 4 we propose the modifications to the basic scheme that are required in order to preserve stationary steadystates, and to keep nonnegative water height (Sect. 4.1). We conclude in Section 5 with a variety of one- and two-dimensional (2D) simulations. Examples of 1D and 2D reconstructions are given in the Appendices.

\section{The Saint-Venant System With a sourCe Term}

In this section we present the model problems which serve as a motivation for the study conducted in this work. We consider a flow in a $1 \mathrm{D}$ channel with a bottom elevation given by $B(x)$. Let $h(x, t)$ represent the fluid depth above the bottom, and $u(x, t)$ be the fluid velocity. The top surface at any time $t$ is therefore given 
by $B(x)+h(x, t)$. The $1 \mathrm{D}$ Saint-Venant equations read

$$
\left\{\begin{array}{l}
h_{t}+(h u)_{x}=0 \\
(h u)_{t}+\left(h u^{2}+\frac{1}{2} g h^{2}\right)_{x}=-g h B_{x}
\end{array}\right.
$$

where $g$ is the gravitational constant and $B_{x}=\mathrm{d} B / \mathrm{d} x$.

In two space dimensions, the velocity is the vector $(u, v)^{T}$, and the shallow water equations take the form of

$$
\left\{\begin{array}{l}
h_{t}+(h u)_{x}+(h v)_{y}=0, \\
(h u)_{t}+\left(h u^{2}+\frac{1}{2} g h^{2}\right)_{x}+(h u v)_{y}=-g h B_{x}, \\
(h v)_{t}+(h u v)_{x}+\left(h v^{2}+\frac{1}{2} g h^{2}\right)_{x}=-g h B_{y} .
\end{array}\right.
$$

Several cases are of special interest:

1. The quasi-stationary state. In the stationary state there is no motion, $u=0$, and the top surface is flat, $h(x, t)+B(x)=C$, for some constant $C$. A quasi-stationary state is generated by slightly perturbing the height of the stationary state. For example, in the 1D case, assume a bottom topography in the shape of a hump and a perturbation of the form $h=C-B(x)+\varepsilon(x)$, where $\varepsilon$ is a compactly supported constant perturbation. In this case the disturbance splits into two waves which, for small $\varepsilon$, are essentially linear propagating at the characteristic speeds $\pm \sqrt{g h}$.

2. A quasi-steady flow. There are steady-states in which the momentum $h u$, is a nonzero constant. The different regimes of such a flow depend on the bottom topography and on the free-stream Froude number $\operatorname{Fr}=u / \sqrt{g h}$. If $\operatorname{Fr}<1$ or $\operatorname{Fr}>1$ everywhere, then the solution is smooth. For intermediate freestream Froude numbers the flow can be transcritical with transitions where Fr passes through 1, and hence one of the eigenvalues, $u \pm \sqrt{g h}$ of the Jacobian, passes through zero. In this case, the steady-state solution can contain a stationary shock.

3. Drain on a bottom. Dry areas, where $h \sim 0$, resemble a state of vacuum in gas dynamics, and are hard to capture. In such areas, even small numerical oscillations may result in negative value(s) of $h$, and then it becomes impossible to calculate the characteristic speeds, $u \pm \sqrt{g h}$. This is the reason why high-order upwind schemes typically fail to compute such solutions.

\section{Central-upwind schemes for Balance laWs}

Before approaching the problem of approximating solutions of $(2.1,2.2)$, we start with the more general problem of approximating solutions for multidimensional-dimensional systems of balance laws of the form,

$$
u_{t}+\nabla_{x} \cdot f(u)=S(u, x, t), \quad x \in \mathbb{R}^{d}, \quad u \in \mathbb{R}^{N},
$$

subject to the initial data, $u(x, 0)=u_{0}(x)$.

\subsection{A one-dimensional scheme}

For simplicity we use a uniform spatial grid $x_{\alpha}:=\alpha \Delta x$, and denote by $\bar{u}_{j}(t)$ the cell average of $u(\cdot, t)$ over the interval $\left(x_{j-\frac{1}{2}}, x_{j+\frac{1}{2}}\right)$,

$$
\bar{u}_{j}(t):=\frac{1}{\Delta x} \int_{x_{j-\frac{1}{2}}}^{x_{j+\frac{1}{2}}} u(x, t) \mathrm{d} x .
$$


An equivalent formulation of (3.1) in terms of cell averages in the 1D case reads

$$
\frac{\mathrm{d}}{\mathrm{d} t} \bar{u}_{j}(t)+\frac{f\left(u\left(x_{j+\frac{1}{2}}, t\right)\right)-f\left(u\left(x_{j-\frac{1}{2}}, t\right)\right)}{\Delta x}=\frac{1}{\Delta x} \int_{x_{j-\frac{1}{2}}}^{x_{j+\frac{1}{2}}} S(u(x, t), x, t) \mathrm{d} x
$$

A semi-discrete scheme is obtained by using an appropriate quadrature for the spatial integral on the RHS of (3.2), and by approximating the fluxes at the half-integer grid points, $x=x_{j \pm \frac{1}{2}}$.

The semi-discrete central-upwind scheme, with an additional term that accounts for the contribution of the source, can be written in the following form,

$$
\frac{\mathrm{d}}{\mathrm{d} t} \bar{u}_{j}(t)=-\frac{H_{j+\frac{1}{2}}(t)-H_{j-\frac{1}{2}}(t)}{\Delta x}+\bar{S}_{j}(t),
$$

where the numerical fluxes, $H_{j+\frac{1}{2}}$, are given by (see [18])

$$
H_{j+\frac{1}{2}}(t):=\frac{a_{j+\frac{1}{2}}^{+} f\left(u_{j+\frac{1}{2}}^{-}\right)-a_{j+\frac{1}{2}}^{-} f\left(u_{j+\frac{1}{2}}^{+}\right)}{a_{j+\frac{1}{2}}^{+}-a_{j+\frac{1}{2}}^{-}}+\frac{a_{j+\frac{1}{2}}^{+} a_{j+\frac{1}{2}}^{-}}{a_{j+\frac{1}{2}}^{+}-a_{j+\frac{1}{2}}^{-}}\left[u_{j+\frac{1}{2}}^{+}-u_{j+\frac{1}{2}}^{-}\right] .
$$

Here, $u_{j+\frac{1}{2}}^{+}:=p_{j+1}\left(x_{j+\frac{1}{2}}, t\right)$ and $u_{j+\frac{1}{2}}^{-}:=p_{j}\left(x_{j+\frac{1}{2}}, t\right)$ are the right and the left values at $x=x_{j+\frac{1}{2}}$ of a conservative, non-oscillatory piecewise polynomial interpolant,

$$
\widetilde{u}(x, t)=\sum_{j} p_{j}(x, t) \chi_{j}
$$

which is reconstructed at each time step from the previously computed cell averages, $\left\{\bar{u}_{j}(t)\right\}$. The pieces $\left\{p_{j}(\cdot, t)\right\}$ are polynomials of a given degree, and $\chi_{j}$ is the characteristic function of the interval $\left[x_{j-1 / 2}, x_{j+1 / 2}\right]$. Note that the order of the piecewise-polynomial reconstruction determines the (formal) order of the numerical fluxes (3.4).

The numerical source term, $\bar{S}_{j}(t)$, requires a special attention and will be treated in Section 4 . Finally, the one-sided local speeds of propagation, $a_{j+\frac{1}{2}}^{ \pm}$, are determined by

$$
\begin{aligned}
& a_{j+\frac{1}{2}}^{+}=\max \left\{\lambda_{N}\left(\frac{\partial f}{\partial u}\left(u_{j+\frac{1}{2}}^{-}\right)\right), \lambda_{N}\left(\frac{\partial f}{\partial u}\left(u_{j+\frac{1}{2}}^{+}\right)\right), 0\right\}, \\
& a_{j+\frac{1}{2}}^{-}=\min \left\{\lambda_{1}\left(\frac{\partial f}{\partial u}\left(u_{j+\frac{1}{2}}^{-}\right)\right), \lambda_{1}\left(\frac{\partial f}{\partial u}\left(u_{j+\frac{1}{2}}^{+}\right)\right), 0\right\},
\end{aligned}
$$

with $\lambda_{1}<\ldots<\lambda_{N}$ being the $N$ eigenvalues of the Jacobian $\partial f / \partial u$.

\section{Remarks.}

1. We would like to emphasize that one of the main advantages of the proposed scheme is its simplicity. Indeed, the 1D system of balance laws can be solved component-wise since no (approximate) Riemann problem solvers were utilized.

2. Since the method we describe in this section is semi-discrete, equations (3.3-3.5) define a system of ODEs. In order to solve this system, one needs to use a stable ODE solver of an appropriate order. In our numerical simulations we have used the third-order strong stability-preserving (SSP) Runge-Kutta solver proposed by Shu et al. in $[9,37,38]$. 
3. In order to construct a central-upwind scheme of a desired order, one needs to use a piecewise polynomial reconstruction, $\left\{p_{j}\right\}$, of an appropriate degree. In the numerical examples below, we have used the secondorder piecewise linear minmod reconstruction and the third-order piecewise quadratic reconstruction, briefly described in Appendix A.

\subsection{A two-dimensional scheme}

We consider the two-dimensional system of balance laws,

$$
u_{t}+f(u)_{x}+g(u)_{y}=S(u, x, y, t)
$$

The cell averages at time $t$,

$$
\bar{u}_{j, k}(t):=\frac{1}{\Delta x \Delta y} \int_{x_{j-\frac{1}{2}}}^{x_{j+\frac{1}{2}}} \int_{y_{k-\frac{1}{2}}}^{y_{k+\frac{1}{2}}} u(x, y, t) \mathrm{d} x \mathrm{~d} y
$$

are evolved using the genuinely multidimensional semi-discrete central-upwind scheme (taken from [18]), with an additional term that counts for the contribution of the source,

$$
\frac{\mathrm{d}}{\mathrm{d} t} \bar{u}_{j, k}(t)=-\frac{H_{j+\frac{1}{2}, k}^{x}(t)-H_{j-\frac{1}{2}, k}^{x}(t)}{\Delta x}-\frac{H_{j, k+\frac{1}{2}}^{y}(t)-H_{j, k-\frac{1}{2}}^{y}(t)}{\Delta y}+\bar{S}_{j, k}(t) .
$$

The fourth-order 2D numerical fluxes, $H^{x}$ and $H^{y}$, are

$$
\begin{aligned}
H_{j+\frac{1}{2}, k}^{x}:= & \frac{a_{j+\frac{1}{2}, k}^{+}}{6\left(a_{j+\frac{1}{2}, k}^{+}-a_{j+\frac{1}{2}, k}^{-}\right)}\left[f\left(u_{j, k}^{N E}\right)+4 f\left(u_{j, k}^{E}\right)+f\left(u_{j, k}^{S E}\right)\right] \\
& -\frac{a_{j+\frac{1}{2}, k}^{-}}{6\left(a_{j+\frac{1}{2}, k}^{+}-a_{j+\frac{1}{2}, k}^{-}\right)}\left[f\left(u_{j+1, k}^{N W}\right)+4 f\left(u_{j+1, k}^{W}\right)+f\left(u_{j+1, k}^{S W}\right)\right] \\
& +\frac{a_{j+\frac{1}{2}, k}^{+} a_{j+\frac{1}{2}, k}^{-}}{6\left(a_{j+\frac{1}{2}, k}^{+}-a_{j+\frac{1}{2}, k}^{-}\right)}\left[u_{j+1, k}^{N W}-u_{j, k}^{N E}+4\left(u_{j+1, k}^{W}-u_{j, k}^{E}\right)+u_{j+1, k}^{S W}-u_{j, k}^{S E}\right]
\end{aligned}
$$

and

$$
\begin{aligned}
H_{j, k+\frac{1}{2}}^{y}:= & \frac{b_{j, k+\frac{1}{2}}^{+}}{6\left(b_{j, k+\frac{1}{2}}^{+}-b_{j, k+\frac{1}{2}}^{-}\right)}\left[g\left(u_{j, k}^{N W}\right)+4 g\left(u_{j, k}^{N}\right)+g\left(u_{j, k}^{N E}\right)\right] \\
& -\frac{b_{j, k+\frac{1}{2}}^{-}}{6\left(b_{j, k+\frac{1}{2}}^{+}-b_{j, k+\frac{1}{2}}^{-}\right)}\left[g\left(u_{j, k+1}^{S W}\right)+4 g\left(u_{j, k+1}^{S}\right)+g\left(u_{j, k+1}^{S E}\right)\right] \\
& +\frac{b_{j, k+\frac{1}{2}}^{+} b_{j, k+\frac{1}{2}}^{-}}{6\left(b_{j, k+\frac{1}{2}}^{+}-b_{j, k+\frac{1}{2}}^{-}\right)}\left[u_{j, k+1}^{S W}-u_{j, k}^{N W}+4\left(u_{j, k+1}^{S}-u_{j, k}^{N}\right)+u_{j, k+1}^{S E}-u_{j, k}^{N E}\right] .
\end{aligned}
$$

The point values in $(3.7,3.8)$ are computed from a non-oscillatory piecewise polynomial reconstruction, $\widetilde{u}(x, y, t)=\sum_{j, k} p_{j, k}(x, y, t) \chi_{j, k}\left(\right.$ where $\chi_{j, k}$ is the characteristic function of the cell $\left.\left[x_{j-\frac{1}{2}}, x_{j+\frac{1}{2}}\right] \times\left[y_{k-\frac{1}{2}}, y_{k+\frac{1}{2}}\right]\right)$, 
and are given by

$$
\begin{gathered}
u_{j, k}^{N}:=p_{j, k}\left(x_{j}, y_{k+\frac{1}{2}}, t\right), u_{j, k}^{S}:=p_{j, k}\left(x_{j}, y_{k-\frac{1}{2}}, t\right), \\
u_{j, k}^{E}:=p_{j, k}\left(x_{j+\frac{1}{2}}, y_{k}, t\right), u_{j, k}^{W}:=p_{j, k}\left(x_{j-\frac{1}{2}}, y_{k}, t\right), u_{j, k}^{N E}:=p_{j, k}\left(x_{j+\frac{1}{2}}, y_{k+\frac{1}{2}}, t\right), \\
u_{j, k}^{N W}:=p_{j, k}\left(x_{j-\frac{1}{2}}, y_{k+\frac{1}{2}}, t\right), u_{j, k}^{S E}:=p_{j, k}\left(x_{j+\frac{1}{2}}, y_{k-\frac{1}{2}}, t\right), u_{j, k}^{S W}:=p_{j, k}\left(x_{j-\frac{1}{2}}, y_{k-\frac{1}{2}}, t\right) .
\end{gathered}
$$

In our numerical simulations, we used the third-order piecewise parabolic reconstruction proposed in [19], which is outlined in Appendix B. This results in a third-order approximation of the fluxes. Similarly to the 1D case, the one-sided local speeds of propagation $a_{j+\frac{1}{2}, k}^{ \pm}$and $b_{j, k+\frac{1}{2}}^{ \pm}$are calculated via

$$
\begin{aligned}
a_{j+\frac{1}{2}, k}^{+} & :=\max \left\{\lambda_{N}\left(\frac{\partial f}{\partial u}\left(u_{j+1, k}^{W}\right)\right), \lambda_{N}\left(\frac{\partial f}{\partial u}\left(u_{j, k}^{E}\right)\right), 0\right\}, \\
b_{j, k+\frac{1}{2}}^{+} & :=\max \left\{\lambda_{N}\left(\frac{\partial g}{\partial u}\left(u_{j, k+1}^{S}\right)\right), \lambda_{N}\left(\frac{\partial g}{\partial u}\left(u_{j, k}^{N}\right)\right), 0\right\}, \\
a_{j+\frac{1}{2}, k}^{-} & :=\min \left\{\lambda_{1}\left(\frac{\partial f}{\partial u}\left(u_{j+1, k}^{W}\right)\right), \lambda_{1}\left(\frac{\partial f}{\partial u}\left(u_{j, k}^{E}\right)\right), 0\right\}, \\
b_{j, k+\frac{1}{2}}^{-} & :=\min \left\{\lambda_{1}\left(\frac{\partial g}{\partial u}\left(u_{j, k+1}^{S}\right)\right), \lambda_{1}\left(\frac{\partial g}{\partial u}\left(u_{j, k}^{N}\right)\right), 0\right\},
\end{aligned}
$$

where $\lambda_{1}(\cdot)<\ldots<\lambda_{N}(\cdot)$ are the eigenvalues of $\partial f / \partial u$ or $\partial g / \partial u$. The numerical source term in (3.6),

$$
\bar{S}_{j, k}(t) \approx \frac{1}{\Delta x \Delta y} \int_{x_{j-\frac{1}{2}}}^{x_{j+\frac{1}{2}}} \int_{y_{k-\frac{1}{2}}}^{y_{k+\frac{1}{2}}} S(u(x, y, t), x, y, t) \mathrm{d} x \mathrm{~d} y
$$

is obtained by an appropriate quadrature, which will be discussed in Section 4 below.

Remark. The generalization of the proposed 2D scheme to higher space dimensions can be derived in a similar manner. We omit the technical details.

\section{Central-upwind schemes for steady states}

In this section we focus on the Saint-Venant equations and derive a specific discretization of the source term that preserves stationary steady-state solutions. For simplicity we fix the gravitational constant $g=1$.

We start with the 1D case and denote the surface of the water at any given time by $w:=h+B$. Noting that $w$, and not $h$, must remain constant when stationary solutions are considered, we rewrite equation (2.1) in terms of $w$ and the momentum $h u$. Since the bottom topography $B(x)$ remains constant in time, we have

$$
\left\{\begin{array}{l}
w_{t}+(h u)_{x}=0 \\
(h u)_{t}+\left[\frac{(h u)^{2}}{w-B}+\frac{1}{2}(w-B)^{2}\right]_{x}=-(w-B) B_{x} .
\end{array}\right.
$$

The same change of variables was done in [35]. 
A similar change of variables can be made in the 2D case. Again, we denote the surface by $w:=h+B$ and rewrite $(2.2)$ in terms of $w, h u$ and $h v$,

$$
\left\{\begin{array}{l}
w_{t}+(h u)_{x}+(h v)_{y}=0 \\
(h u)_{t}+\left[\frac{(h u)^{2}}{w-B}+\frac{1}{2}(w-B)^{2}\right]_{x}+\left[\frac{(h u)(h v)}{w-B}\right]_{y}=-(w-B) B_{x} \\
(h v)_{t}+\left[\frac{(h u)(h v)}{w-B}\right]_{x}+\left[\frac{(h v)^{2}}{w-B}+\frac{1}{2}(w-B)^{2}\right]_{y}=-(w-B) B_{y}
\end{array}\right.
$$

How do we discretize (4.1) and (4.2)? An implementation of (3.3-3.5) in the corresponding 1D case, and of (3.6-3.10) in the 2D case, still requires a quadrature for approximating the averages of the source terms.

In 1D, we are looking for a discretization of $\bar{S}_{j}(t)$ such that stationary steady-state solutions $(u=0, w=$ const) will be preserved by (3.3-3.5). In this case, $\vec{u}_{j+\frac{1}{2}}^{+}=\vec{u}_{j+\frac{1}{2}}^{-}$, where $\vec{u}:=(w, h u)^{T}$. The eigenvalues of the Jacobian are $u \pm \sqrt{h}= \pm \sqrt{w-B}$. If we assume that $w$ is constant, then the one-sided speeds satisfy $a_{j+\frac{1}{2}}^{+}=-a_{j+\frac{1}{2}}^{-}$(given that the point-value of $B\left(x_{j+\frac{1}{2}}\right)$ is known). The numerical flux in (3.4) is then given by

$$
\vec{H}_{j+\frac{1}{2}}=\frac{\vec{f}\left(\vec{u}_{j+\frac{1}{2}}^{+}\right)+\vec{f}\left(\vec{u}_{j+\frac{1}{2}}^{-}\right)}{2}=\vec{f}\left(\vec{u}_{j+\frac{1}{2}}\right), \quad \vec{f}(\vec{u})=\left(\begin{array}{c}
h u \\
\frac{(h u)^{2}}{w-B}+\frac{1}{2}(w-B)^{2}
\end{array}\right) .
$$

The first component of the flux, $H_{j+\frac{1}{2}}^{(1)}$, is identically zero, and the second component is

$$
H_{j+\frac{1}{2}}^{(2)}=\frac{1}{2}\left(w_{j+\frac{1}{2}}-B\left(x_{j+\frac{1}{2}}\right)\right)^{2} .
$$

Since $w_{j+\frac{1}{2}}=w_{j-\frac{1}{2}}=$ Const, the first term on the RHS of (3.3) equals

$$
\begin{aligned}
-\frac{H_{j+\frac{1}{2}}^{(2)}-H_{j-\frac{1}{2}}^{(2)}}{\Delta x} & =-\frac{1}{2 \Delta x}\left(\left(w_{j+\frac{1}{2}}-B\left(x_{j+\frac{1}{2}}\right)\right)^{2}-\left(w_{j-\frac{1}{2}}-B\left(x_{j-\frac{1}{2}}\right)\right)^{2}\right) \\
& =\frac{B\left(x_{j+\frac{1}{2}}\right)-B\left(x_{j-\frac{1}{2}}\right)}{\Delta x} \cdot \frac{w_{j+\frac{1}{2}}-B\left(x_{j+\frac{1}{2}}\right)+w_{j-\frac{1}{2}}-B\left(x_{j-\frac{1}{2}}\right)}{2} .
\end{aligned}
$$

Therefore, in order to preserve stationary steady-states, (4.3) must be canceled by the contribution of the source term. This dictates the following discretization of the average of the source term:

$$
\bar{S}_{j}^{(2)}(t) \approx \frac{1}{\Delta x} \int_{x_{j-\frac{1}{2}}}^{x_{j+\frac{1}{2}}} S^{(2)}(\widetilde{u}(x, t), x, t) \mathrm{d} x \approx-\frac{B\left(x_{j+\frac{1}{2}}\right)-B\left(x_{j-\frac{1}{2}}\right)}{\Delta x} \cdot \frac{\left(w_{j+\frac{1}{2}}^{-}-B\left(x_{j+\frac{1}{2}}\right)\right)+\left(w_{j-\frac{1}{2}}^{+}-B\left(x_{j-\frac{1}{2}}\right)\right)}{2} .
$$

Remark. The quadrature (4.4) is second-order accurate. Therefore, even if we use the third-order numerical fluxes, $H_{j+\frac{1}{2}}$, the resulting scheme is only second-order. At the same time, we would like to stress that such a "mixed"-order scheme seems to provide a much sharper resolution than the "purely"second-order scheme (consult Ex. 1 in Sect. 5). 
In the $2 \mathrm{D}$ case, a similar computation shows that the components of the numerical source term in (3.6) that preserve stationary states are

$$
\begin{aligned}
\bar{S}_{j, k}^{(2)}(t)= & \frac{1}{\Delta x \Delta y} \int_{x_{j-\frac{1}{2}}}^{x_{j+\frac{1}{2}}} \int_{y_{k-\frac{1}{2}}}^{y_{k+\frac{1}{2}}} S^{(2)}(u(x, y, t), x, y, t) \mathrm{d} x \mathrm{~d} y \\
\approx & -\frac{1}{6}\left[\frac{B\left(x_{j+\frac{1}{2}}, y_{k+\frac{1}{2}}\right)-B\left(x_{j-\frac{1}{2}}, y_{k+\frac{1}{2}}\right)}{\Delta x} \cdot \frac{\left(w_{j, k}^{N E}-B\left(x_{j+\frac{1}{2}}, y_{k+\frac{1}{2}}\right)\right)+\left(w_{j, k}^{N W}-B\left(x_{j-\frac{1}{2}}, y_{k+\frac{1}{2}}\right)\right)}{2}\right. \\
& +4 \cdot \frac{B\left(x_{j+\frac{1}{2}}, y_{k}\right)-B\left(x_{j-\frac{1}{2}}, y_{k}\right)}{\Delta x} \cdot \frac{\left(w_{j, k}^{E}-B\left(x_{j+\frac{1}{2}}, y_{k}\right)\right)+\left(w_{j, k}^{W}-B\left(x_{j-\frac{1}{2}}, y_{k}\right)\right)}{2} \\
& \left.+\frac{B\left(x_{j+\frac{1}{2}}, y_{k-\frac{1}{2}}\right)-B\left(x_{j-\frac{1}{2}}, y_{k-\frac{1}{2}}\right)}{\Delta x} \cdot \frac{\left(w_{j, k}^{S E}-B\left(x_{j+\frac{1}{2}}, y_{k-\frac{1}{2}}\right)\right)+\left(w_{j, k}^{S W}-B\left(x_{j-\frac{1}{2}}, y_{k-\frac{1}{2}}\right)\right)}{2}\right],
\end{aligned}
$$

and

$$
\begin{aligned}
\bar{S}_{j, k}^{(3)}(t)= & \frac{1}{\Delta x \Delta y} \int_{x_{j-\frac{1}{2}}}^{x_{j+\frac{1}{2}}} \int_{y_{k-\frac{1}{2}}}^{y_{k+\frac{1}{2}}} S^{(3)}(u(x, y, t), x, y, t) \mathrm{d} x \mathrm{~d} y \\
\approx & -\frac{1}{6}\left[\frac{B\left(x_{j+\frac{1}{2}}, y_{k+\frac{1}{2}}\right)-B\left(x_{j+\frac{1}{2}}, y_{k-\frac{1}{2}}\right)}{\Delta y} \cdot \frac{\left(w_{j, k}^{N E}-B\left(x_{j+\frac{1}{2}}, y_{k+\frac{1}{2}}\right)\right)+\left(w_{j, k}^{S E}-B\left(x_{j+\frac{1}{2}}, y_{k-\frac{1}{2}}\right)\right)}{2}\right. \\
& +4 \cdot \frac{B\left(x_{j}, y_{k+\frac{1}{2}}\right)-B\left(x_{j}, y_{k-\frac{1}{2}}\right)}{\Delta y} \cdot \frac{\left(w_{j, k}^{N}-B\left(x_{j}, y_{k+\frac{1}{2}}\right)\right)+\left(w_{j, k}^{S}-B\left(x_{j}, y_{k-\frac{1}{2}}\right)\right)}{2} \\
& \left.+\frac{B\left(x_{j-\frac{1}{2}}, y_{k+\frac{1}{2}}\right)-B\left(x_{j-\frac{1}{2}}, y_{k-\frac{1}{2}}\right)}{\Delta y} \cdot \frac{\left(w_{j, k}^{N W}-B\left(x_{j-\frac{1}{2}}, y_{k+\frac{1}{2}}\right)\right)+\left(w_{j, k}^{S W}-B\left(x_{j-\frac{1}{2}}, y_{k-\frac{1}{2}}\right)\right)}{2}\right] .
\end{aligned}
$$

\subsection{Central-upwind schemes for dry states}

If one deals with the case when the water height is very small in some areas, then even small numerical oscillations introduced by a piecewise polynomial reconstruction, may lead to negative computed values of $h$. This will make it impossible to compute the one-sided speeds of propagation $a_{j+\frac{1}{2}}^{ \pm}$, and the scheme (3.3-3.5) can not be applied as is. In this section we explain how to avoid such an undesirable situation.

Let us consider the $1 \mathrm{D}$ case (the $2 \mathrm{D}$ case can be treated by a complete analogy). According to (3.3-3.5), the spatial discretization of the first equation in (4.1) gives

$$
\frac{\mathrm{d}}{\mathrm{d} t} \bar{w}_{j}=-\frac{H_{j+\frac{1}{2}}^{(1)}-H_{j-\frac{1}{2}}^{(1)}}{\Delta x}
$$

where

$$
H_{j+\frac{1}{2}}^{(1)}=\frac{a_{j+\frac{1}{2}}^{+}(h u)_{j+\frac{1}{2}}^{-}-a_{j+\frac{1}{2}}^{-}(h u)_{j+\frac{1}{2}}^{+}}{a_{j+\frac{1}{2}}^{+}-a_{j+\frac{1}{2}}^{-}}+\frac{a_{j+\frac{1}{2}}^{+} a_{j+\frac{1}{2}}^{-}}{a_{j+\frac{1}{2}}^{+}-a_{j+\frac{1}{2}}^{-}}\left[w_{j+\frac{1}{2}}^{+}-w_{j+\frac{1}{2}}^{-}\right]
$$


Let us assume that $h \sim 0, B(x)$ is continuous, and $B \not \equiv$ Const in a neighborhood of $x=x_{j+\frac{1}{2}}$. The difference $w_{j+\frac{1}{2}}^{+}-w_{j+\frac{1}{2}}^{-}$must be also $\sim 0$, but this may not be true if the values of $w_{j+\frac{1}{2}}^{ \pm}$are computed from an $r$ th-order piecewise polynomial reconstruction $\widetilde{w}(\cdot, t)$. In such a case, $w_{j+\frac{1}{2}}^{+}-w_{j+\frac{1}{2}}^{-}=C(\Delta x)^{r}, C \sim B^{\prime \prime}\left(x_{j+\frac{1}{2}}\right)$, and therefore, even in the extreme case of $h_{j-1}=h_{j}=h_{j+1}=0$, the nonzero numerical diffusion may lead to negative values of $h$.

Alternatively, one may reconstruct the water height, $\widetilde{h}(\cdot, t)$, in the areas where $h$ is small, and set $w_{j+\frac{1}{2}}^{ \pm}:=$ $h_{j+\frac{1}{2}}^{ \pm}+B\left(x_{j+\frac{1}{2}}\right)$ there. Then $w_{j+\frac{1}{2}}^{+}-w_{j+\frac{1}{2}}^{-}=h_{j+\frac{1}{2}}^{+}-h_{j+\frac{1}{2}}^{-} \sim 0$, and we expect $h$ to remain positive. More precisely, this result is formulated in the following theorem.

Theorem 4.1. Consider the system (4.1) and assume that $B(x)$ is continuous. Consider the central-upwind semi-discrete scheme (3.3-3.5), where the intermediate values $w_{j+\frac{1}{2}}^{ \pm}=h_{j+\frac{1}{2}}^{ \pm}+B\left(x_{j+\frac{1}{2}}\right)$ and $(h u)_{j+\frac{1}{2}}^{ \pm}$are computed from a conservative, monotonicity preserving, TVD reconstruction of the variables $h$ and hu (e.g., the second-order minmod reconstruction (A.2, A.4)). Assume that the resulting system of ODEs is solved with a forward Euler method, and that $\forall j, \bar{h}_{j}^{n}:=\bar{w}_{j}^{n}-\int_{x_{j-\frac{1}{2}}}^{x_{j+\frac{1}{2}}} B(x) \mathrm{d} x \geq 0$. Then $\forall j, \bar{h}_{j}^{n+1} \geq 0$, provided that $\Delta t \leq \frac{\Delta x}{4 a}$, where $a:=\max _{j}\left\{\max \left(a_{j+\frac{1}{2}}^{+},-a_{j+\frac{1}{2}}^{-}\right)\right\}$.

Remark. If instead of the first-order forward Euler method, one uses a higher-order SSP ODE solver (either the Runge-Kutta or the multistep one), the result of Theorem 4.1 is still valid since such ODE solvers can be written as a convex combination of several forward Euler steps, see [9].

Proof. The central-upwind scheme for the first equation in (4.1), together with the reconstruction of $h$ and $h u$, and the forward Euler temporal discretization gives,

$$
\bar{w}_{j}^{n+1}=\bar{w}_{j}^{n}-\lambda\left(H_{j+\frac{1}{2}}^{(1)}-H_{j-\frac{1}{2}}^{(1)}\right), \quad \lambda:=\frac{(\Delta t)^{n}}{\Delta x},
$$

where the numerical flux (4.5) becomes

$$
H_{j+\frac{1}{2}}^{(1)}=\frac{a_{j+\frac{1}{2}}^{+}(h u)_{j+\frac{1}{2}}^{-}-a_{j+\frac{1}{2}}^{-}(h u)_{j+\frac{1}{2}}^{+}}{a_{j+\frac{1}{2}}^{+}-a_{j+\frac{1}{2}}^{-}}+\frac{a_{j+\frac{1}{2}}^{+} a_{j+\frac{1}{2}}^{-}}{a_{j+\frac{1}{2}}^{+}-a_{j+\frac{1}{2}}^{-}}\left[h_{j+\frac{1}{2}}^{+}-h_{j+\frac{1}{2}}^{-}\right] .
$$

If all $\bar{h}_{j}^{n}$ are nonnegative, and if the values of $h_{j+\frac{1}{2}}^{ \pm}$are obtained from a conservative, monotonicity preserving, TVD reconstruction, then $h_{j+\frac{1}{2}}^{ \pm} \geq 0$ and $\bar{h}_{j}^{n}=\left(h_{j-\frac{1}{2}}^{+}+h_{j+\frac{1}{2}}^{-}\right) / 2$ for all $j$, and (4.6) can be rewritten in the following form,

$$
\begin{aligned}
\bar{h}_{j}^{n+1}= & {\left[\frac{1}{2}+\frac{\lambda a_{j-\frac{1}{2}}^{-}}{a_{j-\frac{1}{2}}^{+}-a_{j-\frac{1}{2}}^{-}}\left(a_{j-\frac{1}{2}}^{+}-u_{j-\frac{1}{2}}^{+}\right)\right] h_{j-\frac{1}{2}}^{+}\left[\frac{1}{2}-\frac{\lambda a_{j+\frac{1}{2}}^{+}}{a_{j+\frac{1}{2}}^{+}-a_{j+\frac{1}{2}}^{-}}\left(u_{j+\frac{1}{2}}^{-}-a_{j+\frac{1}{2}}^{-}\right)\right] h_{j+\frac{1}{2}}^{-} } \\
& -\left[\frac{\lambda a_{j+\frac{1}{2}}^{-}}{a_{j+\frac{1}{2}}^{+}-a_{j+\frac{1}{2}}^{-}}\left(a_{j+\frac{1}{2}}^{+}-u_{j+\frac{1}{2}}^{+}\right)\right] h_{j+\frac{1}{2}}^{+}\left[\frac{\lambda a_{j-\frac{1}{2}}^{+}}{a_{j-\frac{1}{2}}^{+}-a_{j-\frac{1}{2}}^{-}}\left(u_{j-\frac{1}{2}}^{-}-a_{j-\frac{1}{2}}^{-}\right)\right] h_{j-\frac{1}{2}}^{-},
\end{aligned}
$$

where $u_{j \pm \frac{1}{2}}^{ \pm}:=(h u)_{j \pm \frac{1}{2}}^{ \pm} / h_{j \pm \frac{1}{2}}^{ \pm}$.

We recall that the local speeds in (4.7) are $a_{j+\frac{1}{2}}^{+}=\max \left(u_{j+\frac{1}{2}}^{+}+\sqrt{h_{j+\frac{1}{2}}^{+}}, u_{j+\frac{1}{2}}^{-}+\sqrt{h_{j+\frac{1}{2}}^{-}}, 0\right)$, and $a_{j+\frac{1}{2}}^{-}=$ $\min \left(u_{j+\frac{1}{2}}^{+}-\sqrt{h_{j+\frac{1}{2}}^{+}}, u_{j+\frac{1}{2}}^{-}-\sqrt{h_{j+\frac{1}{2}}^{-}}, 0\right)$. Therefore, $a_{j+\frac{1}{2}}^{+} \geq 0, a_{j+\frac{1}{2}}^{-} \leq 0, a_{j+\frac{1}{2}}^{+}-u_{j+\frac{1}{2}}^{+} \geq 0, u_{j+\frac{1}{2}}^{-}-a_{j+\frac{1}{2}}^{-} \geq 0 \forall j$, 
and thus the last two terms in (4.8) are nonnegative. The first two terms in (4.8) will be also nonnegative under the CFL restriction $\lambda \leq 1 / 4 a$. Hence, $\bar{h}_{j}^{n+1} \geq 0$ for all $j$, and the proof is completed.

We conclude this section with the description of a high-order central-upwind scheme, suitable for both steadystate and dry state computations:

Algorithm 4.1. Fix a threshold $K>0$. If $\bar{h}_{j-1}^{n}, \bar{h}_{j}^{n}, \bar{h}_{j+1}^{n}>K$, apply the scheme (3.3-3.5) to the system (4.1). Otherwise, replace the first component of the numerical flux, (4.5), with (4.7), and use a conservative, monotonicity preserving, TVD reconstruction for computing the intermediate values of $h_{j \pm \frac{1}{2}}^{ \pm}$and $(h u)_{j \pm \frac{1}{2}}^{ \pm}$.

\section{Remarks.}

1. The resulting scheme does not seem to be sensitive to the choice of the threshold $K$ in Algorithm 4.1. In our numerical experiments we used $K=\max _{j}\left\{\bar{h}_{j}^{0}\right\} / 10$.

2. The CFL condition which guarantees that the values of $h$ remain nonnegative requires time steps $(\Delta t \leq$ $\Delta x / 4 a)$ which are twice smaller than the time steps determined by the usual CFL condition $(\Delta t \leq \Delta x / 2 a)$. We observed that even if the latter CFL number is used, $h$ typically remains nonnegative. In order to make our method more efficient and avoid an increase in the CPU time by a factor of two we have implemented the following strategy:

Assuming that the solution was computed at time $t$, we evolve the solution to time $t+\Delta t$ using CFL $=1 / 2$. If one of the computed values $\bar{h}_{j}(t+\Delta t)$ is negative, the last evolution step is rejected, and instead, the solution is evolved from $t$ to $t+\Delta t / 2$. Note that by Theorem $4.1, \bar{h}_{j}(t+\Delta t / 2) \geq 0$ for all $j$. In practice, our numerical simulations required to reject only a few evolution steps.

3. The $2 \mathrm{D}$ scheme that preserves the positivity of $h$ can be constructed in a similar way. One needs to replace the differences $\left(w_{j+1, k}^{\mathrm{NW}}-w_{j, k}^{\mathrm{NE}}\right), \ldots$ with $\left(h_{j+1, k}^{\mathrm{NW}}-h_{j, k}^{\mathrm{NE}}\right), \ldots$ in the numerical fluxes for the first equation in (4.2), and to reconstruct $\widetilde{h}(\cdot, \cdot, t), \widetilde{h u}(\cdot, \cdot, t)$ and $\widetilde{h v}(\cdot, \cdot, t)$ with the help of a conservative, monotonicity preserving, TVD reconstruction (e.g., the 2D minmod reconstruction (B.3, B.4)) in the areas where $h$ is small.

\section{Numerical Simulations}

In this section we present a variety of $1 \mathrm{D}$ and $2 \mathrm{D}$ examples in which we test our central-upwind methods. In all cases the gravitational constant was taken as $g=1$.

In most examples the CFL number is taken as to satisfy the hyperbolic CFL condition. In the drain problem (Ex. 3), the CFL number is reduced to ensure the stability of the SSP Runge-Kutta solver. One can optimize the choice of the time-step by using more efficient ODE solvers such as the large stability domain Runge-Kutta-type solver DUMKA3 (see [31]) or its recent modifications, ROCK2 or ROCK4 [1,2], available at http://www. unige.ch/math/folks/hairer/software.html.

\section{Example 1 - 1D, small perturbation of a steady-state solution}

The following example is taken from LeVeque [23]. We solve the 1D Saint-Venant system in its original form, (2.1), and in the equivalent form, (4.1). The bottom topography consists of one hump,

$$
B(x)= \begin{cases}0.25(\cos (\pi(x-0.5) / 0.1)+1), & \text { if }|x-0.5|<0.1 \\ 0, & \text { otherwise }\end{cases}
$$

The initial data is the perturbed stationary solution,

$$
w(x, 0)=1+\varepsilon, \quad u(x, 0)=0,
$$

where the perturbation constant $\varepsilon$ is a non-zero constant for $0.1<x<0.2$. We compare the following methods 
for approximating the solutions of this initial-value problem:

1. A "naive" approach in which we solve the system (2.1) in the original variables, $h$ and $h u$. We use the third-order scheme (3.3-3.5) with a Simpson quadrature for the source term.

2. The second-order scheme (3.3-3.5), (4.4) applied to the system (4.1).

3. The third-order scheme (3.3-3.5) with a Simpson quadrature for the source term. Even though this scheme is applied to the system (4.1), it does not preserve stationary steady-state solutions. Due to the high-order treatment of the source term, this method is (formally) third-order accurate.

4. The third-order scheme (3.3-3.5) with the second-order, steady-state preserving discretization of the source term average, (4.4). This method is (formally) second-order accurate.

Figures 5.1-5.2 show the computed surface $w=h+B$ at time $T=0.7$. In all the simulations the number of points was taken as $N=100$. The results are shown for $\varepsilon=10^{-2}, 10^{-5}$. The reference solution is computed by the third-order scheme (3.3-3.5), with the second-order, steady-state preserving method for the source term, (4.4), with $N=1600$ points.

Clearly, the "naive" approach fails to resolve the solution at the vicinity of the hump $(0.4<x<0.6)$, producing an oscillatory solution in this region. These oscillations are magnified as $\varepsilon \rightarrow 0$. The second-order scheme results in a solution which is relatively dissipative though non-oscillatory, as expected. Both third-order methods, which are applied to the modified system (4.1), create a much sharper image of the right-going wave, and in the case of $\varepsilon=10^{-2}$, they seem to produce comparable results (see Fig. 5.1). A big difference is observed in the $\varepsilon=10^{-5}$ case, in which the "purely" third-order method generated oscillations above the hump, while the third-order scheme with the second-order, steady-state preserving method for the source term, is non-oscillatory at that region (see Fig. 5.2). It also produces a much better resolved solution compared with the second-order scheme. This shows that even though this method is only (formally) second-order accurate, the smaller errors in the third-order approximation of the fluxes make a big difference in terms of the resolution of the waves.

\section{Example 2 - Transcritical flow}

We start with the following initial data,

$$
w(x, 0)=1, \quad u(x, 0)=0.3,
$$

where $B(x)$ is given by (5.1). In order to approximate the solution in a finite domain we implement outflow boundary conditions. In this example the flow is transcritical (see Sect. 2). The parameters are chosen such that the free-stream Froude number increases to a value larger than one above the hump and then decreases to less than one, such that a steady-state shock appears on the surface. This problem is taken from [23].

The approximate solution was computed with the third-order scheme (3.3-3.5) with the second-order source term treatment (4.4). The result at time $T=1.8$ with $N=100$ points is presented in Figure 5.3. The solid line represents the reference solution which was computed with $N=1600$ points. Clearly, the steady-state shock is fully resolved already with $N=100$ points.

We would like to emphasize that in [23] LeVeque observed a non-convergence of his Newton iterations at the time when the shock first forms. In order to obtain the solution, he starts with a fractional step method, uses it until the shock forms and an approximate steady-state is reached, and then switches to the quasi-steady method. With the central-upwind scheme we propose in this paper, no special modification is required. The solution presented here is obtained with a direct application of our method to this problem.

\section{Example 3 - Drain on a non-flat bottom}

We solve the system $(4.1,5.1)$ with different bottom topographies and with different initial data. The left boundary condition we assume is a "mirror state"-type condition, and the right boundary condition is an "outlet condition on a dry bed" (consult $[8,11]$ ). This boundary condition on the right side of the domain, allows the water to freely move to the right into the initially dry region. The solution of this initial-boundary value problem at $t \rightarrow \infty$ contains a dry state on the right of the hump. 
(a)

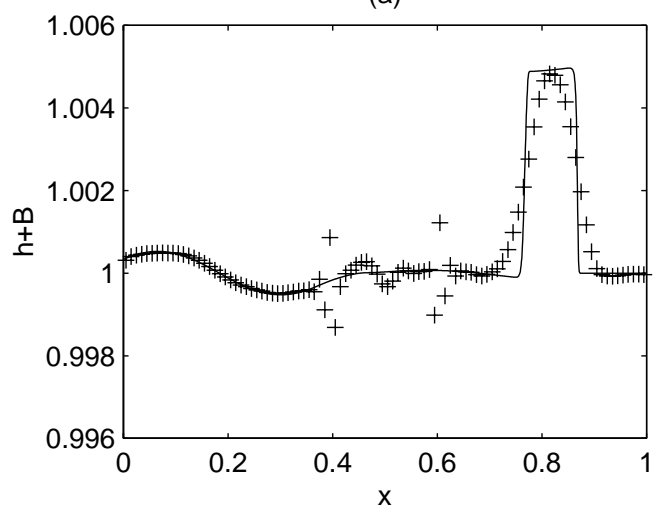

(c)

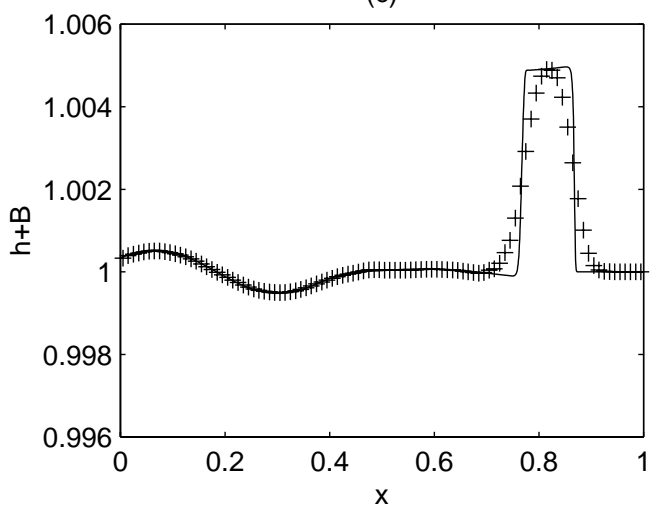

(b)

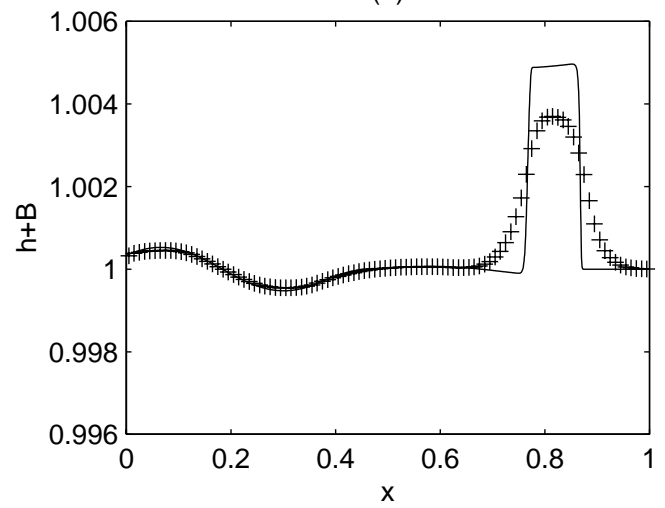

(d)

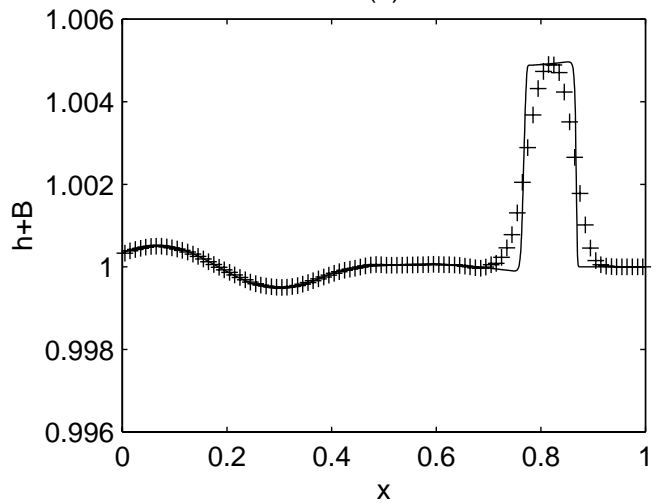

Figure 5.1. $\varepsilon=10^{-2}$. In (a), the solution of $(2.1,5.1,5.2)$ is computed in terms of the original variables $h$ and $u$. In $(\mathrm{b}-\mathrm{d})$, the solution of $(4.1,5.1,5.2)$ is computed in terms of the variables $h+B$ and $h u$. (a) The third-order method with Simpson's rule for the source term. (b) The second-order scheme with the second-order, steady-state preserving method for the source term. (c) The third-order scheme with Simpson's rule for the source term. (d) The third-order scheme with the second-order, steady-state preserving method for the source term.

In Figures 5.4-5.5 we show the solution obtained with the initial data

$$
w(x, 0)=0.8, \quad u(x, 0)=0,
$$

and the bottom (5.1). The solution was computed using $N=100$ grid points and is shown at times $T=$ $0.5,0.75,1,3,50$. The variables that are plotted in these figures are $w=h+B$ and $h u$. As expected the solution converges to a steady-state solution in which water exists only on the left hand side of the hump. In Figures 5.65.7 we show the solution obtained with the same initial data (5.4) and a two-hump bottom which is plotted in a dashed line. Here we compute the solution with $N=200$ points and present it at times $T=0.5,1,3,5,50$.

In Figures 5.8-5.11 we use a different two-humps bottom $B(x)$. We use two sets of initial data: for the simulations we present in Figures 5.8-5.9 the initial data is taken as

$$
w(x, 0)=\left\{\begin{array}{ll}
1.5, & x<0.6, \\
0, & \text { otherwise },
\end{array} \quad u(x, 0)=0\right.
$$


(a)

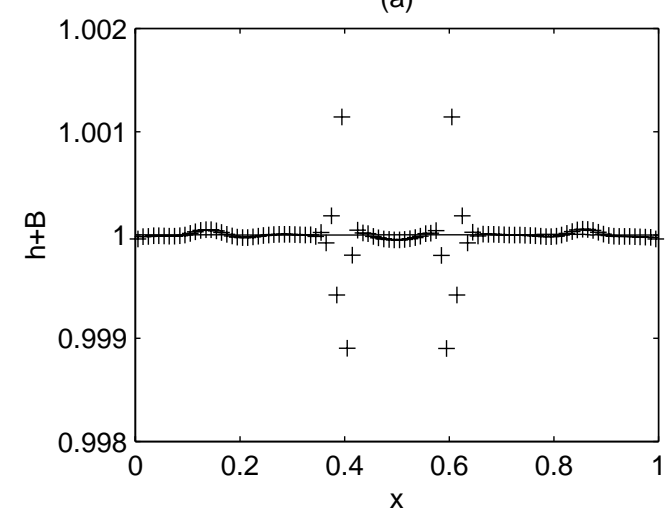

(c)

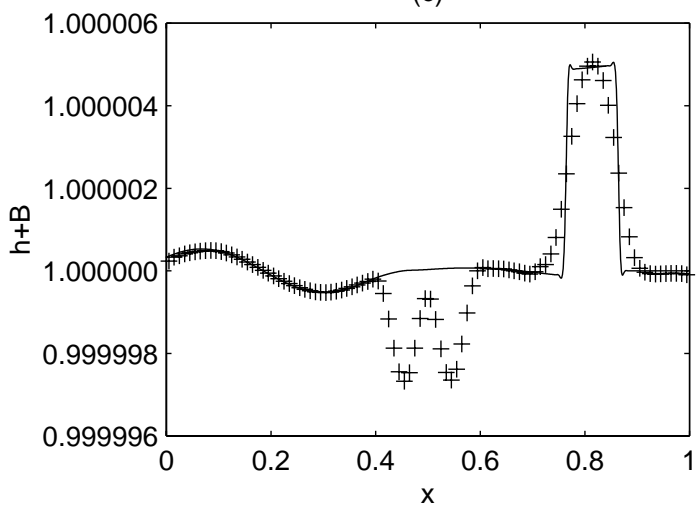

(b)

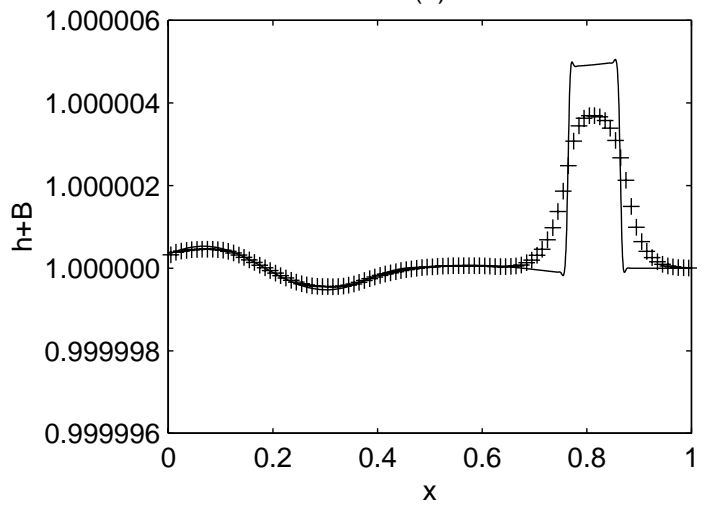

(d)

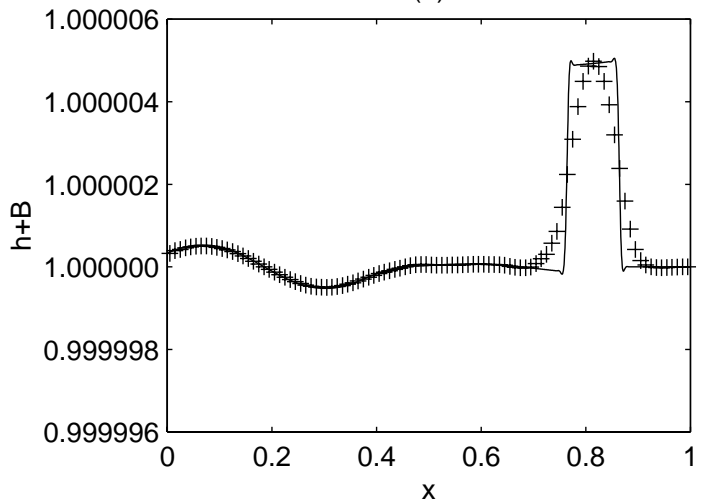

Figure 5.2. $\varepsilon=10^{-5}$. In (a), the solution of $(2.1,5.1,5.2)$ is computed in terms of the original variables $h$ and $u$. In $(\mathrm{b}-\mathrm{d})$, the solution of $(4.1,5.1,5.2)$ is computed in terms of the variables $h+B$ and $h u$. (a) The third-order method with Simpson's rule for the source term. (b) The second-order scheme with the second-order, steady-state preserving method for the source term. (c) The third-order scheme with Simpson's rule for the source term. (d) The third-order scheme with the second-order, steady-state preserving method for the source term.

while in Figures 5.10-5.11 the initial data is

$$
w(x, 0)=\left\{\begin{array}{ll}
1.5, & x<0.2, \\
0, & \text { otherwise },
\end{array} \quad u(x, 0)=0 .\right.
$$

Here we observe a qualitatively different behavior in the solution. While in the first case (5.5), the water remains in both reservoirs, in the second case (5.6) where less water is taken above the right reservoir, the water escapes out of the right reservoir and the steady-state solution includes water only in the left one.

\section{Example 4 - 2D, small perturbation of a steady-state solution}

In this example, taken from LeVeque [23], we solve the $2 \mathrm{D}$ equations $(4.2)$ in the domain $[0,2] \times[0,1]$. The bottom consists of an elliptical shaped hump

$$
B(x, y)=0.8 \exp \left(-5(x-0.9)^{2}-50(y-0.5)^{2}\right) .
$$




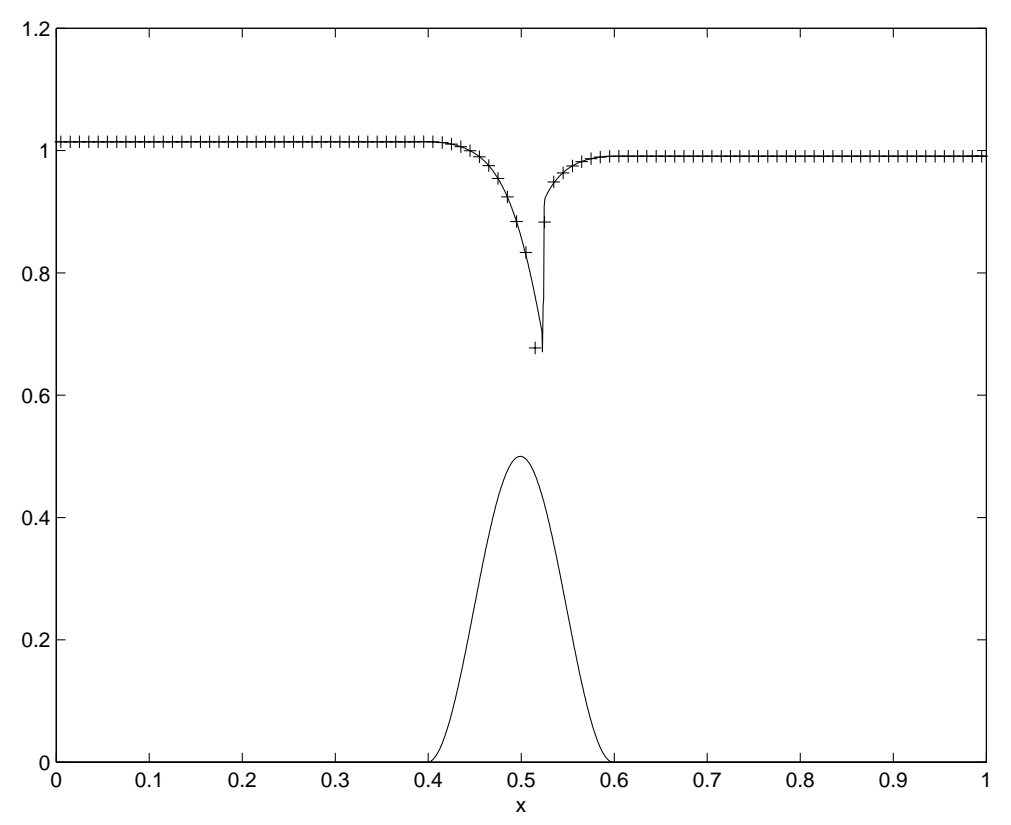

Figure 5.3. A stationary shock in the transcritical case with the initial data (5.3).

The surface is initially flat with $w(x, y, 0)=1$ except for $0.05<x<0.15$ where $\varepsilon=0.01$ is added to $w$. Figure 5.12 displays the right-going disturbance as it propagates past the hump, on a $200 \times 100$ and a $600 \times$ 300 grids. The surface of the water, $w(x, y, t)$, is presented at times $T=0.6,0.9,1.2,1.5,1.8$. Clearly, our simple central-upwind scheme is capable of resolving the complex small features of the flow (compare with [23]).

\section{Appendix A: Non-oscillatory pieCEWISE POLYNOMial RECONSTRUCTIONS}

A major ingredient in the construction of Godunov-type schemes for conservation laws is a piecewise polynomial reconstruction. Typically, second-order schemes require a piecewise linear reconstruction $[22,32]$. Thirdorder schemes employ a piecewise quadratic approximation and one of the possibilities is to use an essentially non-oscillatory (ENO) reconstruction. In the 1D case we refer the reader to $[14,37]$. Taking a convex combination of interpolants that are reconstructed on different stencils results in a reduction in the order of the polynomial. This leads to the Weighted ENO (WENO) interpolants $[25,26,29]$. For multidimensional Central WENO (CWENO) schemes we refer to [26]. The ENO-type reconstructions require a measure on the smoothness of the reconstruction, which in turn requires certain a-priori information about the solution. One-dimensional non-oscillatory piecewise quadratic reconstructions which do not require the use of smoothness indicators were proposed in $[19,28,30]$. Two-dimensional generalizations of these reconstructions were presented in $[19,33]$.

We give a brief description of the 1D third-order non-oscillatory reconstruction from [19] which we used in our numerical experiments. We denote by $D_{ \pm}$and $D_{0}$ the one-sided and the central divided differences

$$
D_{ \pm} v(x):= \pm \frac{v(x \pm \Delta x)-v(x)}{\Delta x}, \quad D_{0} v(x):=\frac{v(x+\Delta x)-v(x-\Delta x)}{2 \Delta x},
$$

and consider the basic piecewise quadratic reconstruction

$$
q_{j}^{n}(x)=\left(\bar{u}_{j}^{n}-\frac{(\Delta x)^{2}}{24} D_{+} D_{-} \bar{u}_{j}^{n}\right)+D_{0} \bar{u}_{j}^{n}\left(x-x_{j}\right)+\frac{1}{2} D_{+} D_{-} \bar{u}_{j}^{n}\left(x-x_{j}\right)^{2}, \quad x \in\left(x_{j-\frac{1}{2}}, x_{j+\frac{1}{2}}\right),
$$


$\mathrm{T}=0.5$
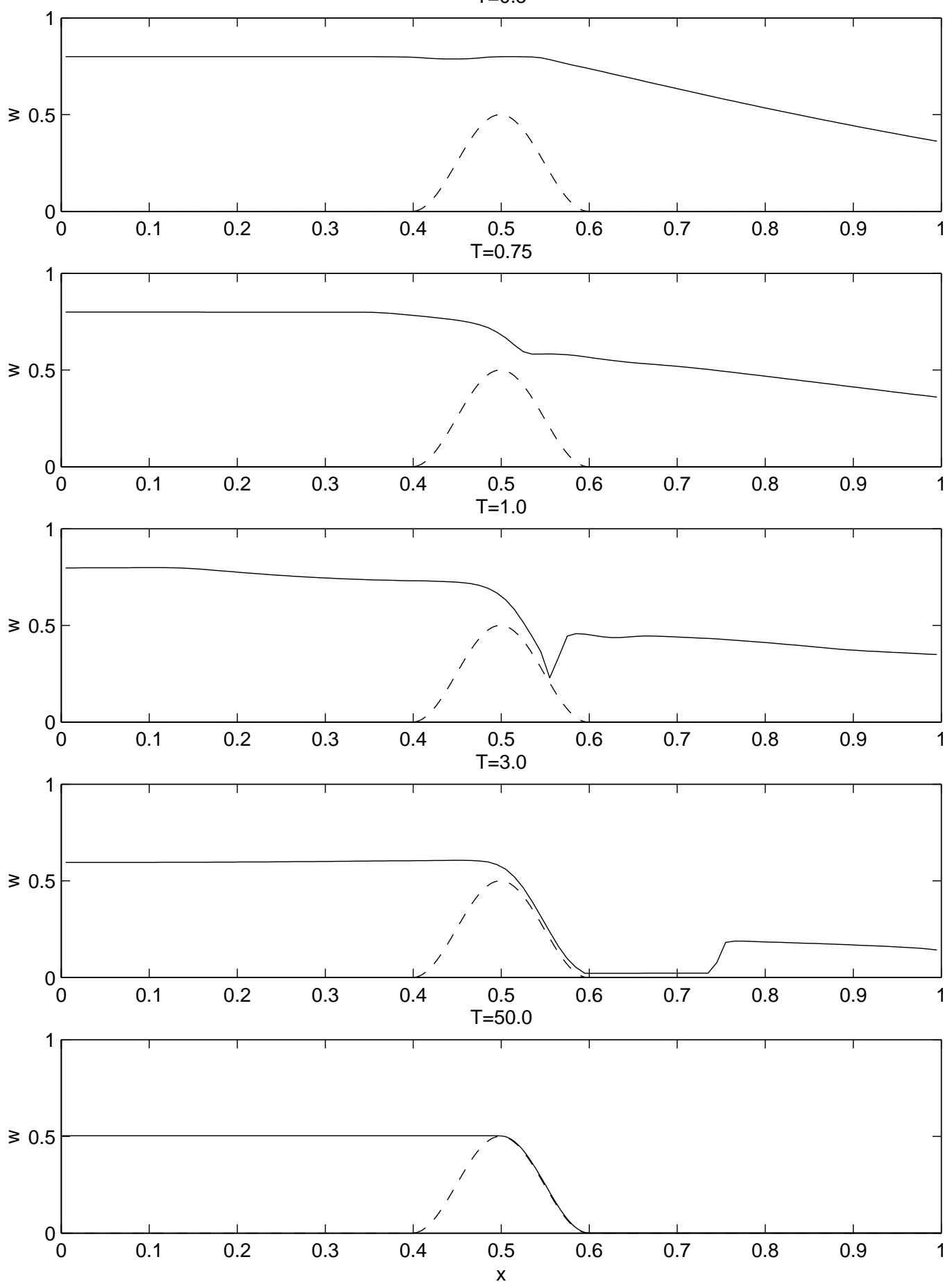

Figure 5.4. Drain on a non-flat bottom with the initial data (5.4) and the bottom (5.1). $N=100$. 

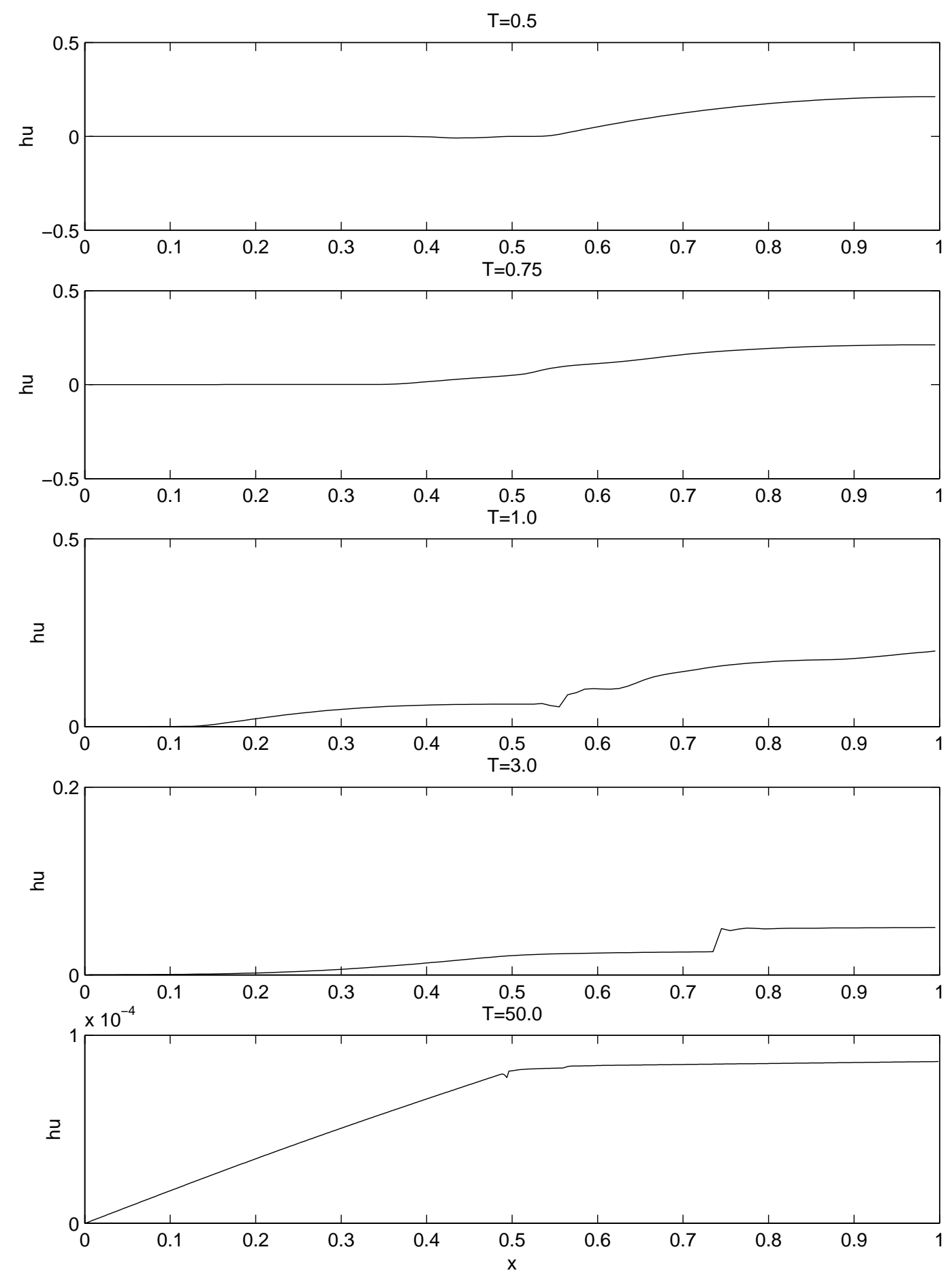

Figure 5.5. Drain on a non-flat bottom with the initial data (5.4) and the bottom (5.1). $N=100$. 
$\mathrm{T}=0.5$
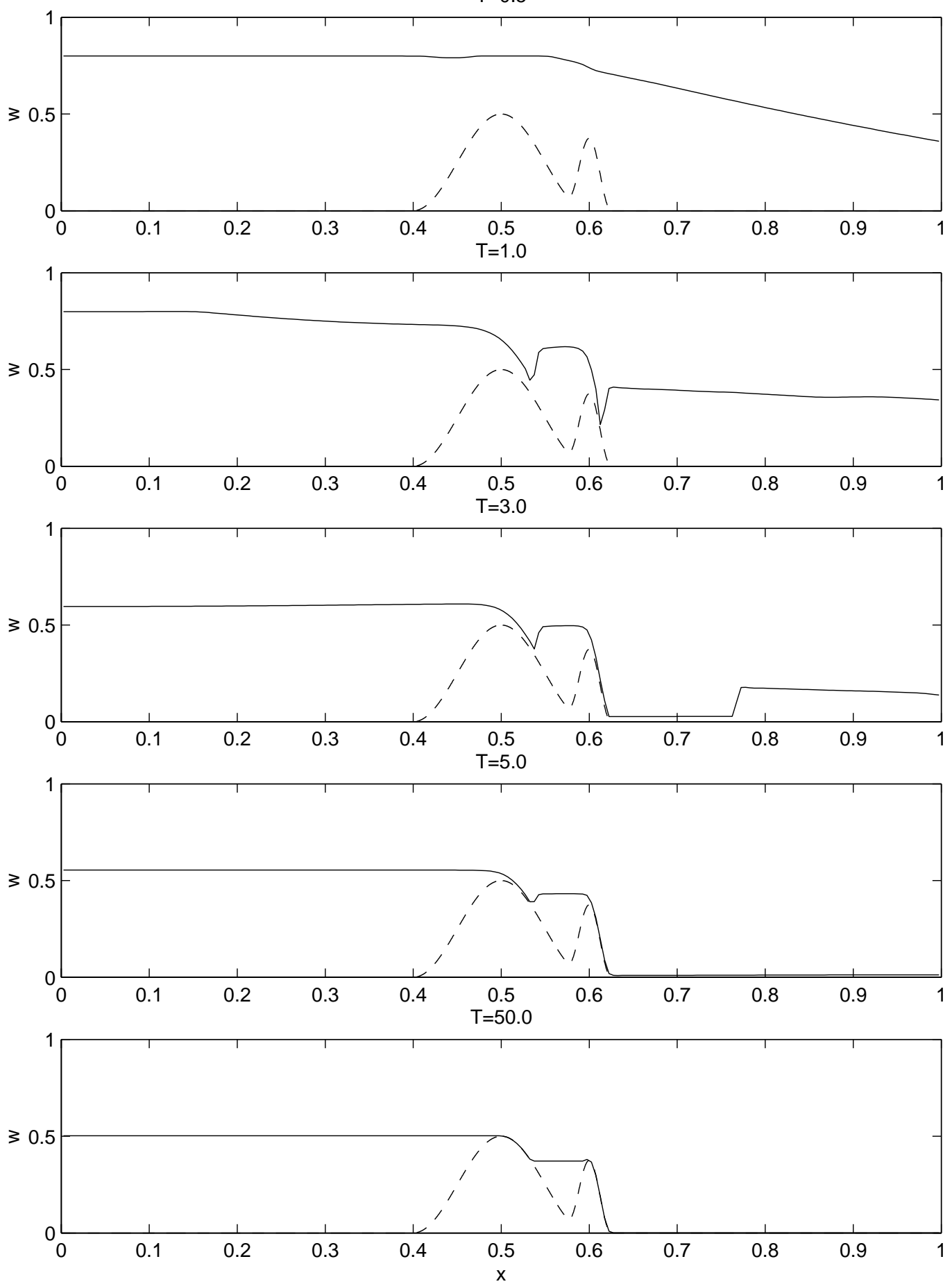

Figure 5.6. Drain on a non-flat bottom with the initial data (5.4). $N=200$. 

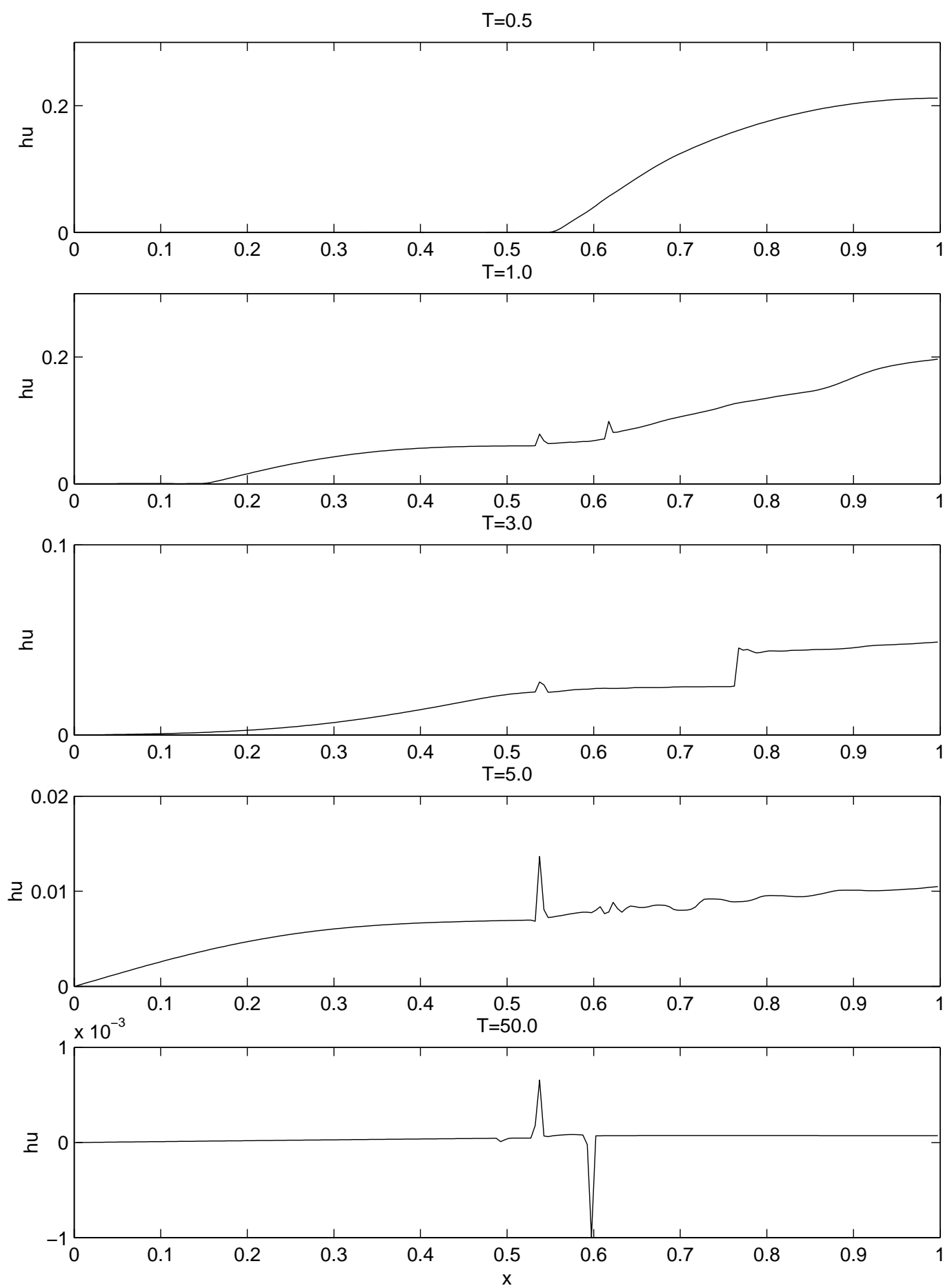

Figure 5.7. Drain on a non-flat bottom with the initial data (5.4). $N=200$. 
$\mathrm{T}=0.5$
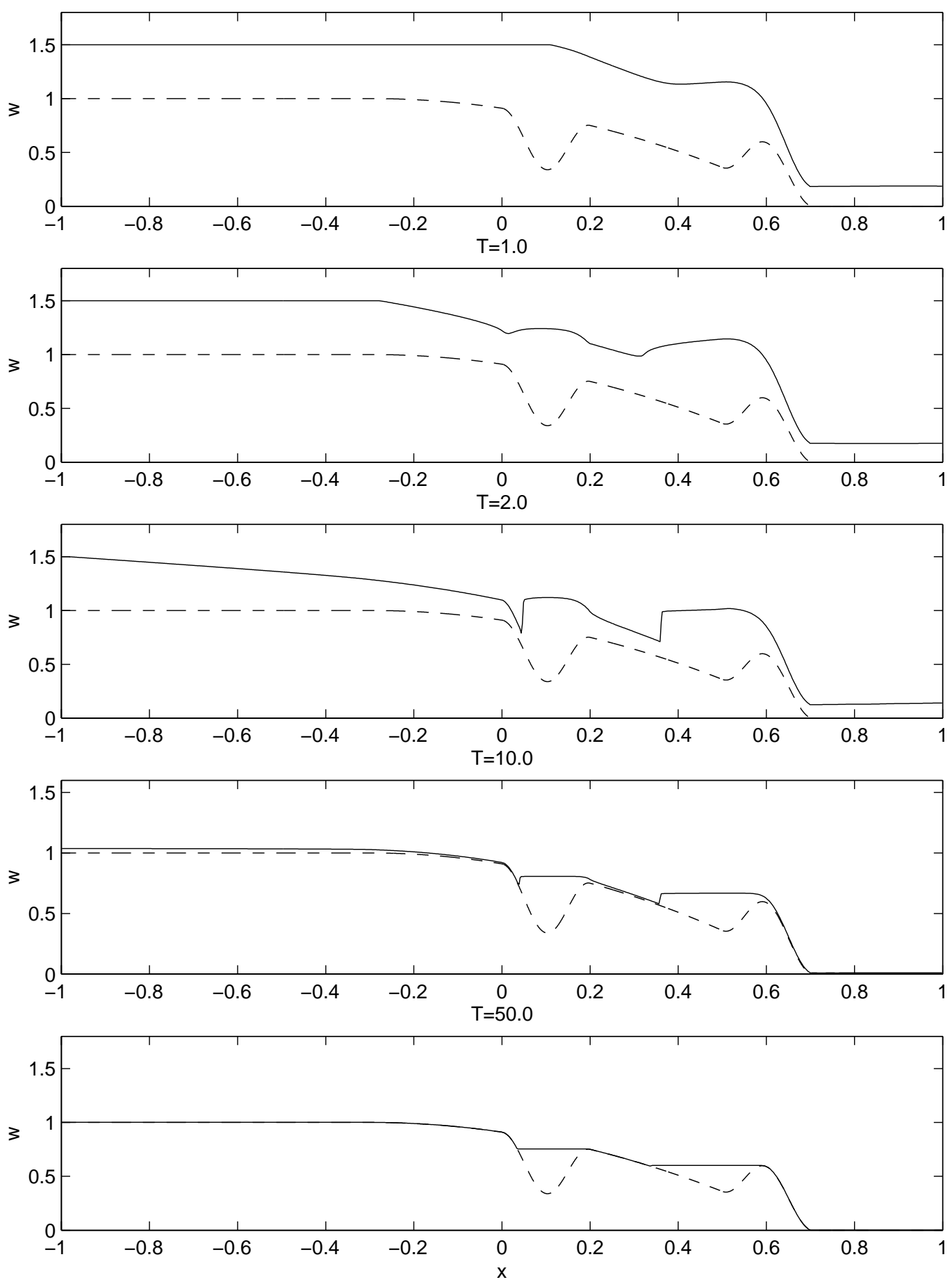

FiguRE 5.8. Drain on a non-flat bottom with the initial data (5.5). $N=800$. 

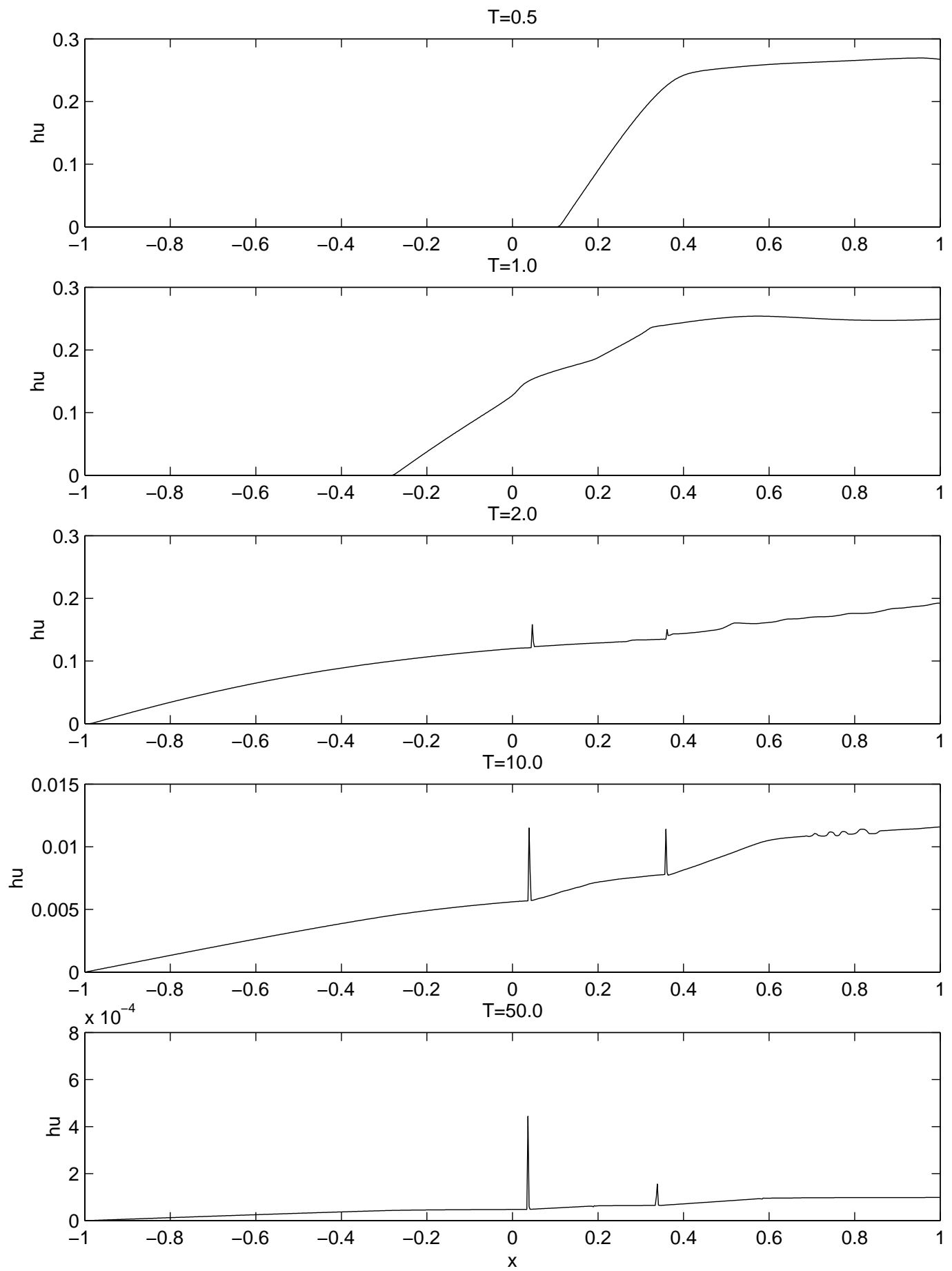

Figure 5.9. Drain on a non-flat bottom with the initial data (5.5). $N=800$. 
$\mathrm{T}=0.2$
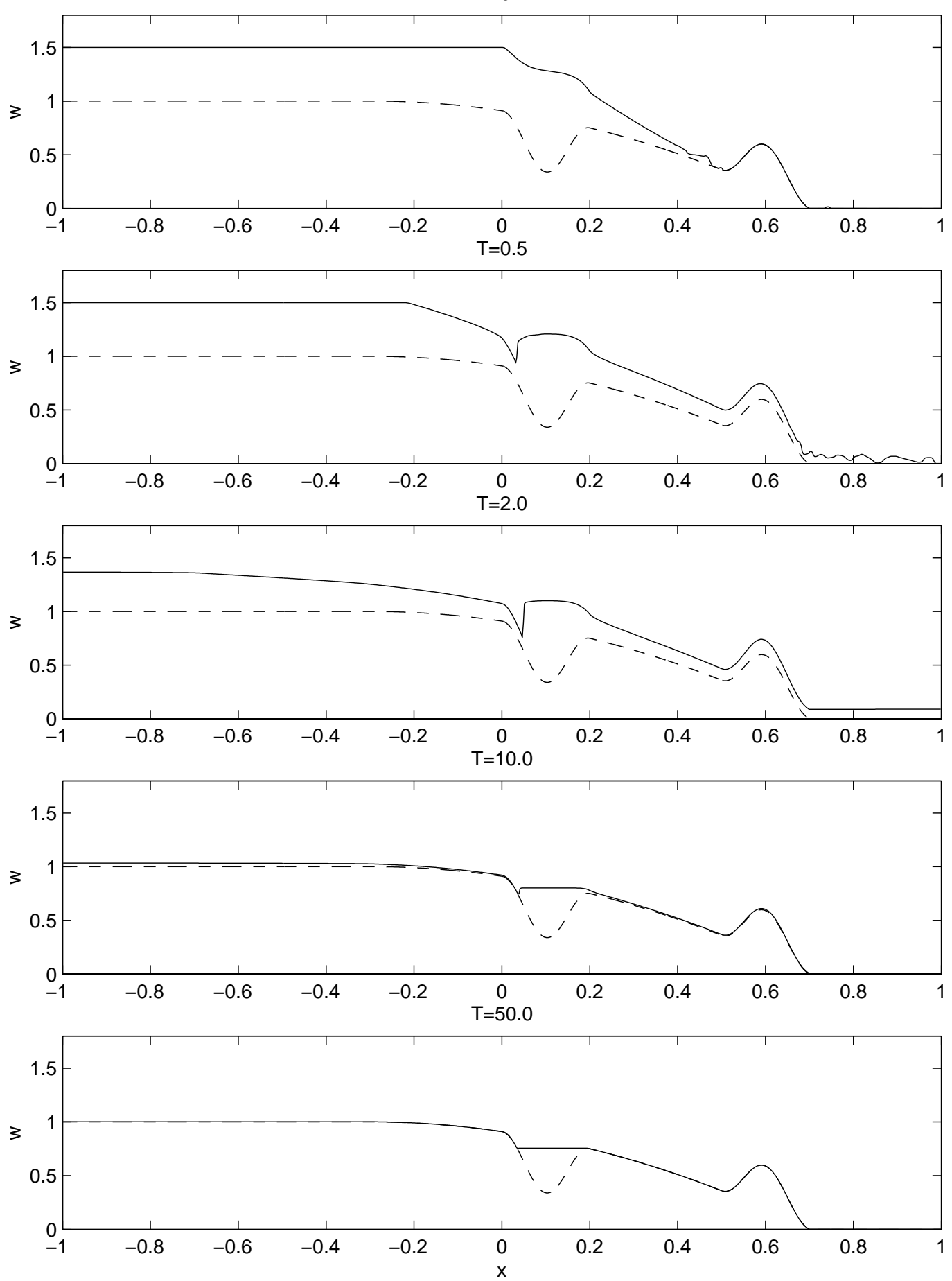

Figure 5.10. Drain on a non-flat bottom with the initial data (5.6). $N=800$. 

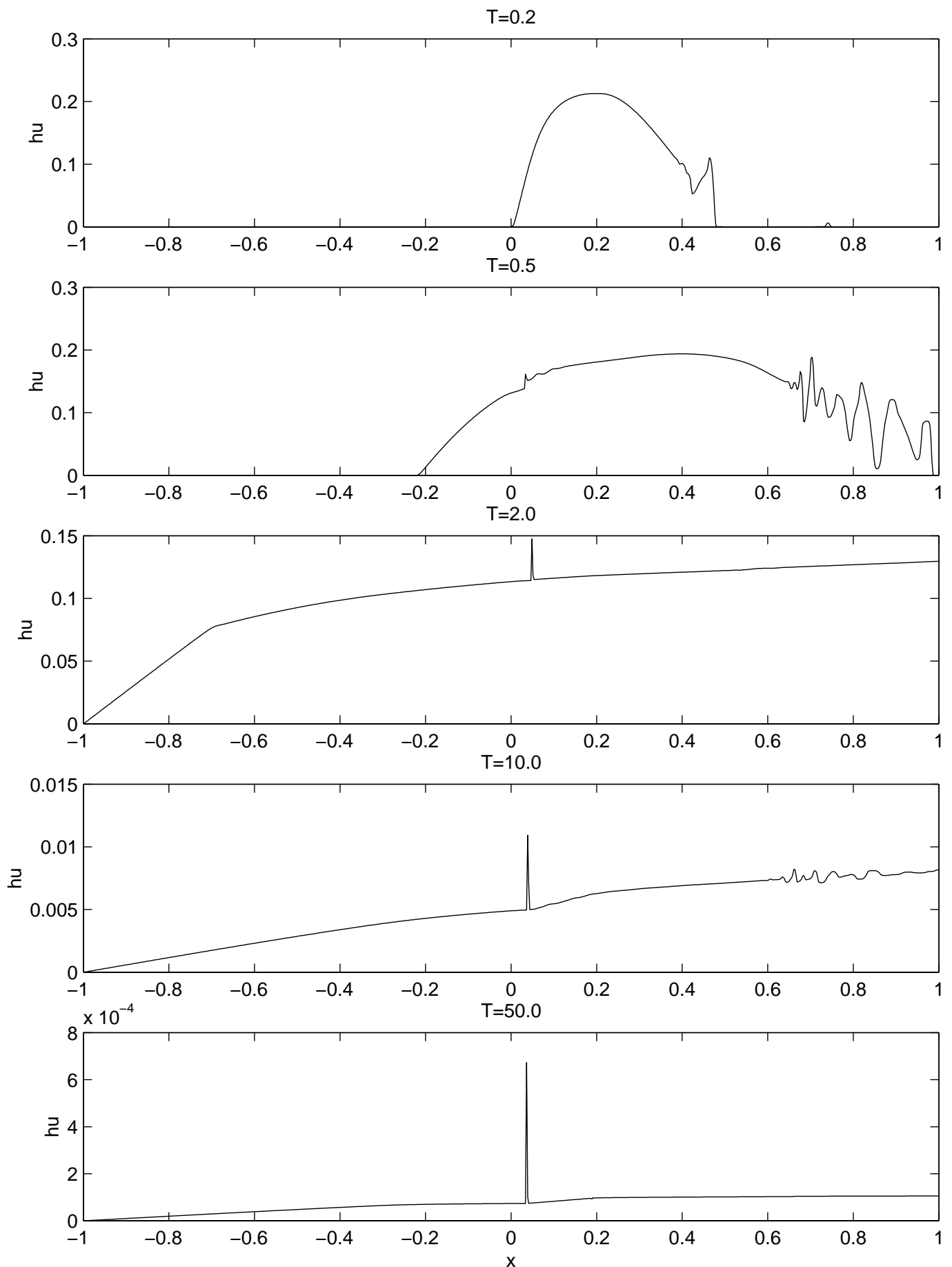

FiguRE 5.11. Drain on a non-flat bottom with the initial data (5.6). $N=800$. 

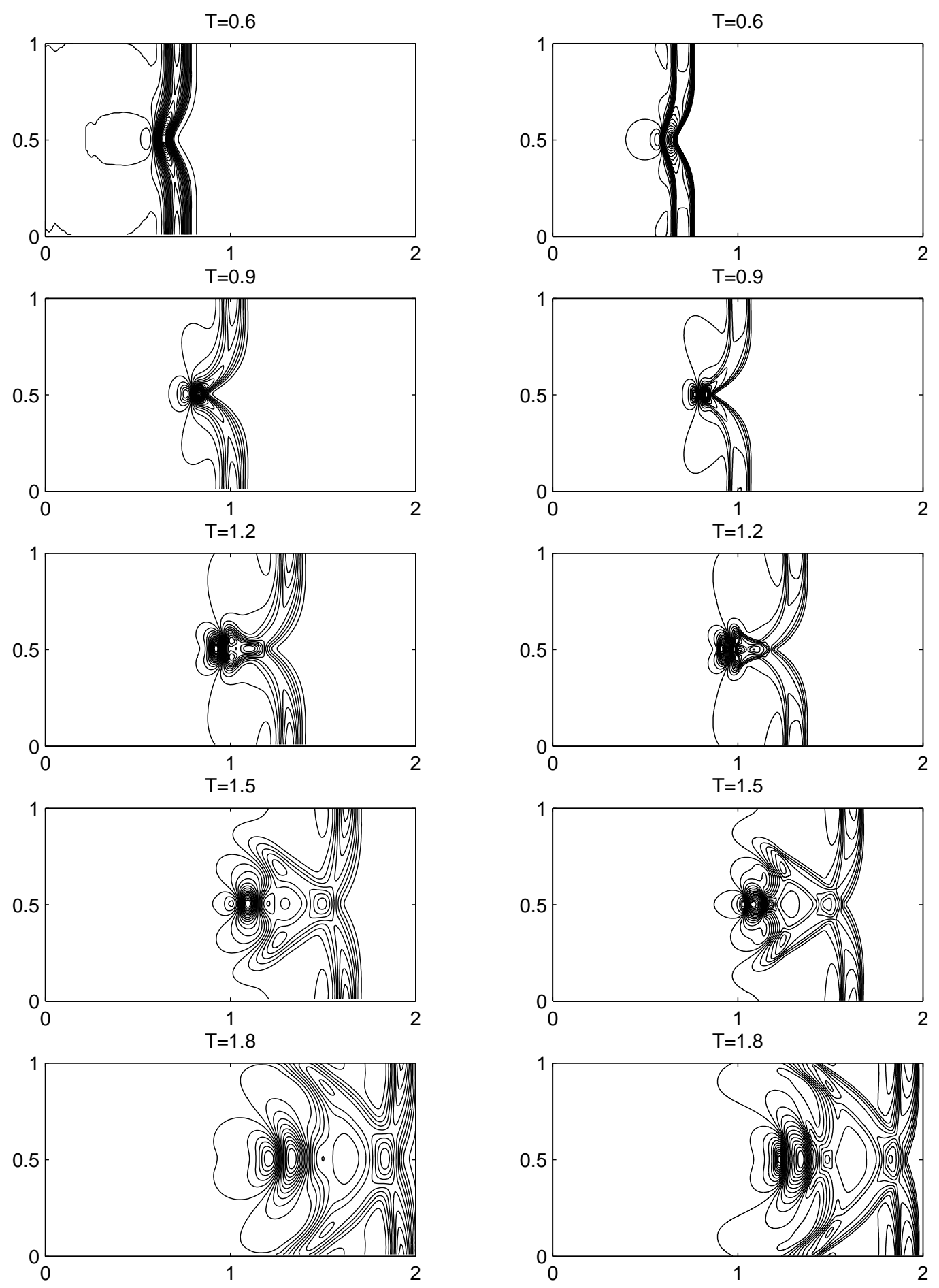

Figure 5.12. The solution of $(4.2,5.7)$. The images on the left side of the figure were computed on a $200 \times 100$ grid. The images on the right side of the figure were computed on a $600 \times 300$ grid. 
which is conservative, accurate, and satisfies a shape-preserving property (for details see [19,30]). In general, this reconstruction is oscillatory since new extrema may be created at the interface points $\left\{x_{j+\frac{1}{2}}\right\}$. To prevent this, a convex combination of the basic parabolas $q_{j}^{n}$ and the piecewise linear interpolant $L_{j}^{n}$ is used, namely

$$
p_{j}^{n}(x)=\left(1-\theta_{j}^{n}\right) L_{j}^{n}(x)+\theta_{j}^{n} q_{j}^{n}(x), \quad 0<\theta_{j}^{n}<1,
$$

where

$$
L_{j}^{n}(x)=\bar{u}_{j}^{n}+s_{j}^{n}\left(x-x_{j}\right) .
$$

An appropriate choice of $\left\{\theta_{j}^{n}\right\}$ and $\left\{s_{j}^{n}\right\}$ guarantees that the new piecewise quadratic reconstruction $\left\{p_{j}^{n}\right\}$ is non-oscillatory [19]. We take $\left\{\theta_{j}^{n}\right\}$ to be

$$
\theta_{j}^{n}:=\left\{\begin{array}{lc}
\min \left\{\frac{M_{j+\frac{1}{2}}^{n}-L_{j}^{n}\left(x_{j+\frac{1}{2}}\right)}{M_{j}^{n}-L_{j}^{n}\left(x_{j+\frac{1}{2}}\right)}, \frac{m_{j-\frac{1}{2}}^{n}-L_{j}^{n}\left(x_{j-\frac{1}{2}}\right)}{m_{j}^{n}-L_{j}^{n}\left(x_{j-\frac{1}{2}}\right)}, 1\right\}, & \text { if } \bar{u}_{j-1}^{n}<\bar{u}_{j}^{n}<\bar{u}_{j+1}^{n}, \\
\min \left\{\frac{M_{j-\frac{1}{2}}^{n}-L_{j}^{n}\left(x_{j-\frac{1}{2}}\right)}{M_{j}^{n}-L_{j}^{n}\left(x_{j-\frac{1}{2}}\right)}, \frac{m_{j+\frac{1}{2}}^{n}-L_{j}^{n}\left(x_{j+\frac{1}{2}}\right)}{m_{j}^{n}-L_{j}^{n}\left(x_{j+\frac{1}{2}}\right)}, 1\right\}, & \text { if } \bar{u}_{j-1}^{n}>\bar{u}_{j}^{n}>\bar{u}_{j+1}^{n}, \\
1, & \text { otherwise, }
\end{array}\right.
$$

where

$$
M_{j}^{n}=\max \left\{q_{j}^{n}\left(x_{j+\frac{1}{2}}\right), q_{j}^{n}\left(x_{j-\frac{1}{2}}\right)\right\}, \quad m_{j}^{n}=\min \left\{q_{j}^{n}\left(x_{j+\frac{1}{2}}\right), q_{j}^{n}\left(x_{j-\frac{1}{2}}\right)\right\}
$$

and

$$
\begin{gathered}
M_{j \pm \frac{1}{2}}^{n}=\max \left\{\frac{1}{2}\left(L_{j}^{n}\left(x_{j \pm \frac{1}{2}}\right)+L_{j \pm 1}^{n}\left(x_{j \pm \frac{1}{2}}\right)\right), q_{j \pm 1}^{n}\left(x_{j \pm \frac{1}{2}}\right)\right\} \\
m_{j \pm \frac{1}{2}}^{n}=\min \left\{\frac{1}{2}\left(L_{j}^{n}\left(x_{j \pm \frac{1}{2}}\right)+L_{j \pm 1}^{n}\left(x_{j \pm \frac{1}{2}}\right)\right), q_{j \pm 1}^{n}\left(x_{j \pm \frac{1}{2}}\right)\right\} .
\end{gathered}
$$

Our choice of the non-oscillatory piecewise linear approximation $\left\{L_{j}^{n}\right\}$ is the standard minmod interpolant (A.2) (see $[22,32,40])$ with

$$
s_{j}^{n}=\operatorname{minmod}\left(\frac{\bar{u}_{j}^{n}-\bar{u}_{j-1}^{n}}{\Delta x}, \frac{\bar{u}_{j+1}^{n}-\bar{u}_{j}^{n}}{\Delta x}\right),
$$

where

$$
\operatorname{minmod}(a, b):=\frac{\operatorname{sgn}(a)+\operatorname{sgn}(b)}{2} \min (|a|,|b|) .
$$

If one sets all the slopes $s_{j}^{n}$ to be zero, then (A.1-A.3) is reduced to the original reconstruction from [30]. The final piecewise parabolic reconstruction is

$$
p_{j}^{n}(x)=\left(\bar{u}_{j}^{n}-\theta_{j}^{n} \frac{(\Delta x)^{2}}{24} D_{+} D_{-} \bar{u}_{j}^{n}\right)+\left(\theta_{j}^{n} D_{0} \bar{u}_{j}^{n}+\left(1-\theta_{j}^{n}\right) s_{j}^{n}\right)\left(x-x_{j}\right)+\frac{\theta_{j}^{n}}{2} D_{+} D_{-} \bar{u}_{j}^{n}\left(x-x_{j}\right)^{2},
$$

where $\theta_{j}^{n}$ and $s_{j}^{n}$ are given by (A.3) and (A.4), respectively. 
Remark. Our second-order central-upwind scheme in Section 5, employs a piecewise linear reconstruction (A.2) with slopes that are computed by a one-parameter family of generalized minmod limiters $[32,39,40]$

$$
s_{j}^{n}=\operatorname{minmod}\left(\theta \frac{\bar{u}_{j}^{n}-\bar{u}_{j-1}^{n}}{\Delta x}, \frac{\bar{u}_{j+1}^{n}-\bar{u}_{j-1}^{n}}{2 \Delta x}, \theta \frac{\bar{u}_{j+1}^{n}-\bar{u}_{j}^{n}}{\Delta x}\right) .
$$

Here, $\theta \in[1,2]$, and the multivariate minmod function is defined by

$$
\operatorname{minmod}\left(x_{1}, x_{2}, \ldots\right):= \begin{cases}\min _{j}\left\{x_{j}\right\}, & \text { if } x_{j}>0 \forall j \\ \max _{j}\left\{x_{j}\right\}, & \text { if } x_{j}<0 \forall j, \\ 0, & \text { otherwise. }\end{cases}
$$

Notice that larger $\theta$ 's in (A.6) correspond to less dissipative but more oscillatory limiters $[32,39,40]$. Our numerical experiments indicate that the optimal value is about $\theta \approx 1.3$.

\section{Appendix B: An example of a tWo-Dimensional, third-ORder, PIECEWISE QUADRATIC RECONSTRUCTION}

In this section we briefly describe the 2D third-order piecewise quadratic reconstruction [19], we used in our numerical examples. It can be viewed as a generalization of (A.5). The main idea is to use a convex combination of the (possibly) oscillatory basic parabolas and non-oscillatory linear functions in order to construct a thirdorder non-oscillatory interpolant. The resulting piecewise quadratic reconstruction $\left\{p_{j, k}^{n}\right\}$ is

$$
p_{j, k}^{n}(x, y)=\left(1-\theta_{j, k}^{n}\right) L_{j, k}^{n}(x, y)+\theta_{j, k}^{n} q_{j, k}^{n}(x, y), \quad 0<\theta_{j, k}^{n}<1 .
$$

Here, $\left\{q_{j, k}^{n}\right\}$ is the basic parabola

$$
\begin{aligned}
q_{j, k}^{n}(x, y)= & \left(\bar{u}_{j, k}^{n}-\frac{(\Delta x)^{2}}{24} D_{+}^{x} D_{-}^{x} \bar{u}_{j, k}^{n}-\frac{(\Delta y)^{2}}{24} D_{+}^{y} D_{-}^{y} \bar{u}_{j, k}^{n}\right)+D_{0}^{x} \bar{u}_{j, k}^{n}\left(x-x_{j}\right)+D_{0}^{y} \bar{u}_{j, k}^{n}\left(y-y_{k}\right)+ \\
& +\frac{1}{2} D_{+}^{x} D_{-}^{x} \bar{u}_{j, k}^{n}\left(x-x_{j}\right)^{2}+D_{0}^{x} D_{0}^{y} \bar{u}_{j, k}^{n}\left(x-x_{j}\right)\left(y-y_{k}\right)+\frac{1}{2} D_{+}^{y} D_{-}^{y} \bar{u}_{j, k}^{n}\left(y-y_{k}\right)^{2}
\end{aligned}
$$

where we use the notation

$$
\begin{gathered}
D_{ \pm}^{x} v(x, y):= \pm \frac{v(x \pm \Delta x, y)-v(x, y)}{\Delta x}, \quad D_{ \pm}^{y} v(x, y):= \pm \frac{v(x, y \pm \Delta y)-v(x, y)}{\Delta y}, \\
D_{0}^{x} v(x, y):=\frac{v(x+\Delta x, y)-v(x-\Delta x, y)}{2 \Delta x}, \quad D_{0}^{y} v(x, y):=\frac{v(x, y+\Delta y)-v(x, y-\Delta y)}{2 \Delta y} .
\end{gathered}
$$

The linear functions $\left\{L_{j, k}^{n}\right\}$ are given by

$$
L_{j, k}^{n}(x, y)=\bar{u}_{j, k}^{n}+s_{j, k}^{x}\left(x-x_{j}\right)+s_{j, k}^{y}\left(y-y_{k}\right),
$$

where the slopes $\left\{s_{j, k}^{x}, s_{j, k}^{y}\right\}$ are computed using the minmod limiter,

$$
s_{j, k}^{x}=\operatorname{minmod}\left(\frac{\bar{u}_{j, k}^{n}-\bar{u}_{j-1, k}^{n}}{\Delta x}, \frac{\bar{u}_{j+1, k}^{n}-\bar{u}_{j, k}^{n}}{\Delta x}\right), s_{j, k}^{y}=\operatorname{minmod}\left(\frac{\bar{u}_{j, k}^{n}-\bar{u}_{j, k-1}^{n}}{\Delta y}, \frac{\bar{u}_{j, k+1}^{n}-\bar{u}_{j, k}^{n}}{\Delta y}\right) .
$$

For our new scheme, only eight point values need to be computed in each $(j, k)$-cell: $u_{j, k}^{\mathrm{N}}, u_{j, k}^{\mathrm{E}}, u_{j, k}^{\mathrm{S}}, u_{j, k}^{\mathrm{W}}, u_{j, k}^{\mathrm{NE}}$, $u_{j, k}^{\mathrm{NW}}, u_{j, k}^{\mathrm{SE}}$, and $u_{j, k}^{\mathrm{SW}}$. They may be calculated using the "dimension-by-dimension" approach which we apply in 
two steps. First, we use the reconstruction (B.1, B.2) with

$$
\theta_{j, k}^{n}=\min \left\{\theta_{j, k}^{x}, \theta_{j, k}^{y}\right\},
$$

to approximate the point values in the coordinate directions $-u_{j, k}^{\mathrm{N}}, u_{j, k}^{\mathrm{E}}, u_{j, k}^{\mathrm{S}}$ and $u_{j, k}^{\mathrm{W}}$. Here, the limiters $\left\{\theta_{j, k}^{x}\right\}$ and $\left\{\theta_{j, k}^{y}\right\}$ are determined by (A.3) with

$$
L_{j}^{n}(\cdot)=L_{j, k}^{n}\left(\cdot, y_{k}\right), \quad q_{j}(\cdot)=q_{j, k}^{n}\left(\cdot, y_{k}\right),
$$

and

$$
L_{j}^{n}(\cdot)=L_{j, k}^{n}\left(x_{k}, \cdot\right), \quad q_{j}^{n}(\cdot)=L_{j, k}^{n}\left(x_{k}, \cdot\right) .
$$

To ensure that there are no oscillations in the diagonal directions, the point values $u_{j, k}^{\mathrm{NE}}, u_{j, k}^{\mathrm{NW}}, u_{j, k}^{\mathrm{SE}}$ and $u_{j, k}^{\mathrm{SW}}$ are computed by another reconstruction which is determined by

$$
\hat{p}_{j, k}^{n}(x, y)=\left(1-\hat{\theta}_{j, k}^{n}\right) \hat{L}_{j, k}^{n}(x, y)+\hat{\theta}_{j, k}^{n} \hat{q}_{j, k}^{n}(x, y), \quad 0<\hat{\theta}_{j, k}^{n}<1 .
$$

The adjusted basic parabolas $\left\{\hat{q}_{j, k}^{n}\right\}$ are

$$
\begin{aligned}
\hat{q}_{j, k}^{n}(x, y)= & \bar{u}_{j, k}^{n}-\frac{\Delta^{2}}{48}\left(D_{+}^{d^{+}} D_{-}^{d^{+}} \bar{u}_{j, k}^{n}+D_{+}^{d^{-}} D_{-}^{d^{-}} \bar{u}_{j, k}^{n}\right)+\frac{\Delta}{2} D_{0}^{d^{+}} \bar{u}_{j, k}^{n}\left[\frac{y-y_{k}}{\Delta y}+\frac{x-x_{j}}{\Delta x}\right] \\
& +\frac{\Delta}{2} D_{0}^{d^{-}} \bar{u}_{j, k}^{n}\left[\frac{y-y_{k}}{\Delta y}-\frac{x-x_{j}}{\Delta x}\right]+\frac{\Delta^{2}}{4} D_{0}^{d^{+}} D_{0}^{d^{-}} \bar{u}_{j, k}^{n}\left[\left(\frac{y-y_{k}}{\Delta y}\right)^{2}-\left(\frac{x-x_{j}}{\Delta x}\right)^{2}\right] \\
& +\frac{\Delta^{2}}{8} D_{+}^{d^{+}} D_{-}^{d^{+}} \bar{u}_{j, k}^{n}\left[\frac{y-y_{k}}{\Delta y}+\frac{x-x_{j}}{\Delta x}\right]^{2}+\frac{\Delta^{2}}{8} D_{+}^{d^{-}} D_{-}^{d^{-}} \bar{u}_{j, k}^{n}\left[\frac{y-y_{k}}{\Delta y}-\frac{x-x_{j}}{\Delta x}\right]^{2},
\end{aligned}
$$

where $\Delta:=\sqrt{(\Delta x)^{2}+(\Delta y)^{2}}$, and the divided differences in the diagonal directions are

$$
\begin{aligned}
& D_{ \pm}^{d^{+}} v(x, y):= \pm \frac{v(x \pm \Delta x, y \pm \Delta y)-v(x, y)}{\Delta} \\
& D_{0}^{d^{+}} v(x, y):=\frac{v(x+\Delta x, y+\Delta y)-v(x-\Delta x, y-\Delta y)}{2 \Delta}, \\
& D_{ \pm}^{d^{-}} v(x, y):= \pm \frac{v(x \mp \Delta x, y \pm \Delta y)-v(x, y)}{\Delta} \\
& D_{0}^{d^{-}} v(x, y):=\frac{v(x-\Delta x, y+\Delta y)-v(x+\Delta x, y-\Delta y)}{2 \Delta} .
\end{aligned}
$$

The corresponding linear functions $\left\{\hat{L}_{j, k}^{n}\right\}$ are given by

$$
\hat{L}_{j, k}^{n}(x, y)=\bar{u}_{j, k}^{n}+\frac{\Delta}{2}\left\{\hat{s}_{j, k}^{+}\left[\frac{y-y_{k}}{\Delta y}+\frac{x-x_{j}}{\Delta x}\right]+\hat{s}_{j, k}^{-}\left[\frac{y-y_{k}}{\Delta y}-\frac{x-x_{j}}{\Delta x}\right]\right\},
$$

where the slopes are computed using the minmod limiter, applied in the diagonal directions

$$
\begin{aligned}
& \hat{s}_{j, k}^{+}=\operatorname{minmod}\left(\frac{\bar{u}_{j, k}^{n}-\bar{u}_{j-1, k-1}^{n}}{\Delta}, \frac{\bar{u}_{j+1, k+1}^{n}-\bar{u}_{j, k}^{n}}{\Delta}\right), \\
& \hat{s}_{j, k}^{-}=\operatorname{minmod}\left(\frac{\bar{u}_{j, k}^{n}-\bar{u}_{j+1, k-1}^{n}}{\Delta}, \frac{\bar{u}_{j-1, k+1}^{n}-\bar{u}_{j, k}^{n}}{\Delta}\right) .
\end{aligned}
$$


The parameter $\hat{\theta}_{j, k}^{n}$ in (B.6) is determined by

$$
\hat{\theta}_{j, k}^{n}=\min \left\{\hat{\theta}_{j, k}^{+}, \hat{\theta}_{j, k}^{-}\right\}
$$

where $\hat{\theta}_{j, k}^{+}$and $\hat{\theta}_{j, k}^{-}$are computed similarly to (A.3), namely

$$
\hat{\theta}_{j, k}^{ \pm}:=\left\{\begin{array}{r}
\min \left\{\frac{M_{j \pm \frac{1}{2}, k+\frac{1}{2}}^{ \pm}-\hat{L}_{j, k}^{n}\left(x_{j \pm \frac{1}{2}}, y_{k+\frac{1}{2}}\right)}{M_{j, k}^{ \pm}-\hat{L}_{j, k}^{n}\left(x_{j \pm \frac{1}{2}}, y_{k+\frac{1}{2}}\right)}, \frac{m_{j \mp \frac{1}{2}, k-\frac{1}{2}}^{ \pm}-\hat{L}_{j, k}^{n}\left(x_{j \mp \frac{1}{2}}, y_{k-\frac{1}{2}}\right)}{m_{j, k}^{ \pm}-\hat{L}_{j, k}^{n}\left(x_{j \mp \frac{1}{2}}, y_{k-\frac{1}{2}}\right)}, 1\right\} \\
\min \left\{\frac{M_{j \mp \frac{1}{2}, k-\frac{1}{2}}^{ \pm}-\hat{L}_{j, k}^{n}\left(x_{j \mp \frac{1}{2}}, y_{k-\frac{1}{2}}\right)}{M_{j, k}^{ \pm}-\hat{L}_{j, k}^{n}\left(x_{j \mp \frac{1}{2}}, y_{k-\frac{1}{2}}\right)}, \frac{m_{j \pm \frac{1}{2}, k+\frac{1}{2}}^{ \pm}-\hat{L}_{j, k}^{n}\left(x_{j \pm \frac{1}{2}}, y_{k+\frac{1}{2}}\right)}{m_{j, k}^{ \pm}-\hat{L}_{j, k}^{n}\left(x_{j \pm \frac{1}{2}}, y_{k+\frac{1}{2}}\right)}, 1\right\} \\
\text { if } \bar{u}_{j \mp 1, k-1}^{n}>\bar{u}_{j \pm 1, k+1}^{n}>\bar{u}_{j \pm 1, k+1}^{n}, \\
\text { otherwise },
\end{array}\right.
$$

where

$$
M_{j, k}^{ \pm}=\max \left\{\hat{q}_{j, k}^{n}\left(x_{j \pm \frac{1}{2}}, y_{k+\frac{1}{2}}\right), \hat{q}_{j, k}^{n}\left(x_{j \mp \frac{1}{2}}, y_{k-\frac{1}{2}}\right)\right\}, \quad m_{j, k}^{ \pm}=\min \left\{\hat{q}_{j, k}^{n}\left(x_{j \pm \frac{1}{2}}, y_{k+\frac{1}{2}}\right), \hat{q}_{j, k}^{n}\left(x_{j \mp \frac{1}{2}}, y_{k-\frac{1}{2}}\right)\right\}
$$

and

$$
\begin{aligned}
& M_{j \pm \frac{1}{2}, k \pm \frac{1}{2}}^{+}=\max \left\{\frac{1}{2}\left(\hat{L}_{j, k}^{n}\left(x_{j \pm \frac{1}{2}}, y_{k \pm \frac{1}{2}}\right)+\hat{L}_{j \pm 1, k \pm 1}^{n}\left(x_{j \pm \frac{1}{2}}, y_{k \pm \frac{1}{2}}\right)\right), \hat{q}_{j \pm 1, k \pm 1}^{n}\left(x_{j \pm \frac{1}{2}}, y_{k \pm \frac{1}{2}}\right)\right\} \\
& m_{j \pm \frac{1}{2}, k \pm \frac{1}{2}}^{+}=\min \left\{\frac{1}{2}\left(\hat{L}_{j, k}^{n}\left(x_{j \pm \frac{1}{2}}, y_{k \pm \frac{1}{2}}\right)+\hat{L}_{j \pm 1, k \pm 1}^{n}\left(x_{j \pm \frac{1}{2}}, y_{k \pm \frac{1}{2}}\right)\right), \hat{q}_{j \pm 1, k \pm 1}^{n}\left(x_{j \pm \frac{1}{2}}, y_{k \pm \frac{1}{2}}\right)\right\} \\
& M_{j \mp \frac{1}{2}, k \pm \frac{1}{2}}^{-}=\max \left\{\frac{1}{2}\left(\hat{L}_{j, k}^{n}\left(x_{j \mp \frac{1}{2}}, y_{k \pm \frac{1}{2}}\right)+\hat{L}_{j \mp 1, k \pm 1}^{n}\left(x_{j \mp \frac{1}{2}}, y_{k \pm \frac{1}{2}}\right)\right), \hat{q}_{j \mp 1, k \pm 1}^{n}\left(x_{j \mp \frac{1}{2}}, y_{k \pm \frac{1}{2}}\right)\right\}, \\
& m_{j \mp \frac{1}{2}, k \pm \frac{1}{2}}^{-}=\min \left\{\frac{1}{2}\left(\hat{L}_{j, k}^{n}\left(x_{j \mp \frac{1}{2}}, y_{k \pm \frac{1}{2}}\right)+\hat{L}_{j \mp 1, k \pm 1}^{n}\left(x_{j \mp \frac{1}{2}}, y_{k \pm \frac{1}{2}}\right)\right), \hat{q}_{j \mp 1, k \pm 1}^{n}\left(x_{j \mp \frac{1}{2}}, y_{k \pm \frac{1}{2}}\right)\right\} .
\end{aligned}
$$

In summary, the point values $u_{j, k}^{\mathrm{N}}, u_{j, k}^{\mathrm{S}}, u_{j, k}^{\mathrm{E}}$ and $u_{j, k}^{\mathrm{W}}$ are computed as the corresponding values of

$$
\begin{aligned}
p_{j, k}^{n}(x, y)= & \left(\bar{u}_{j, k}^{n}-\theta_{j, k}^{n} \frac{(\Delta x)^{2}}{24} D_{+}^{x} D_{-}^{x} \bar{u}_{j, k}^{n}-\theta_{j, k}^{n} \frac{(\Delta y)^{2}}{24} D_{+}^{y} D_{-}^{y} \bar{u}_{j, k}^{n}\right) \\
& +\left(\theta_{j, k}^{n} D_{0}^{x} \bar{u}_{j, k}^{n}+\left(1-\theta_{j, k}^{n}\right) s_{j, k}^{x}\right)\left(x-x_{j}\right)+\left(\theta_{j, k}^{n} D_{0}^{y} \bar{u}_{j, k}^{n}+\left(1-\theta_{j, k}^{n}\right) s_{j, k}^{y}\right)\left(y-y_{k}\right) \\
& +\frac{\theta_{j, k}^{n}}{2} D_{+}^{x} D_{-}^{x} \bar{u}_{j, k}^{n}\left(x-x_{j}\right)^{2}+\theta_{j, k}^{n} D_{0}^{x} D_{0}^{y} \bar{u}_{j, k}^{n}\left(x-x_{j}\right)\left(y-y_{k}\right)+\frac{\theta_{j, k}^{n}}{2} D_{+}^{y} D_{-}^{y} \bar{u}_{j, k}^{n}\left(y-y_{k}\right)^{2},
\end{aligned}
$$


where $\theta_{j, k}^{n}, s_{j, k}^{x}$ and $s_{j, k}^{y}$ are given by (B.5) and (B.4), respectively, and the corner values $u_{j, k}^{\mathrm{NE}}, u_{j, k}^{\mathrm{NW}}, u_{j, k}^{\mathrm{SE}}$ and $u_{j, k}^{\mathrm{SW}}$ are obtained from

$$
\begin{aligned}
\hat{p}_{j, k}^{n}(x, y)= & \left(\bar{u}_{j, k}^{n}-\hat{\theta}_{j, k}^{n} \frac{\Delta^{2}}{48}\left(D_{+}^{d^{+}} D_{-}^{d^{+}}+D_{+}^{d^{-}} D_{-}^{d^{-}}\right) \bar{u}_{j, k}^{n}\right) \\
& +\frac{\Delta}{2 \Delta x}\left(\hat{\theta}_{j, k}^{n}\left(D_{0}^{d^{+}}-D_{0}^{d^{-}}\right) \bar{u}_{j, k}^{n}+\left(1-\hat{\theta}_{j, k}^{n}\right)\left(\hat{s}_{j, k}^{+}-\hat{s}_{j, k}^{-}\right)\right)\left(x-x_{j}\right) \\
& +\frac{\Delta}{2 \Delta y}\left(\hat{\theta}_{j, k}^{n}\left(D_{0}^{d^{+}}+D_{0}^{d^{-}}\right) \bar{u}_{j, k}^{n}+\left(1-\hat{\theta}_{j, k}^{n}\right)\left(\hat{s}_{j, k}^{+}+\hat{s}_{j, k}^{-}\right)\right)\left(y-y_{k}\right) \\
& +\hat{\theta}_{j, k}^{n} \frac{\Delta^{2}}{8(\Delta x)^{2}}\left(D_{+}^{d^{+}} D_{-}^{d^{+}}-2 D_{0}^{d^{+}} D_{0}^{d^{-}}+D_{+}^{d^{-}} D_{-}^{d^{-}}\right) \bar{u}_{j, k}^{n}\left(x-x_{j}\right)^{2} \\
& +\hat{\theta}_{j, k}^{n} \frac{\Delta^{2}}{4 \Delta x \Delta y}\left(D_{+}^{d^{+}} D_{-}^{d^{+}}-D_{+}^{d^{-}} D_{-}^{d^{-}}\right) \bar{u}_{j, k}^{n}\left(x-x_{j}\right)\left(y-y_{k}\right)+ \\
& +\hat{\theta}_{j, k}^{n} \frac{\Delta^{2}}{8(\Delta y)^{2}}\left(D_{+}^{d^{+}} D_{-}^{d^{+}}+2 D_{0}^{d^{+}} D_{0}^{d^{-}}+D_{+}^{d^{-}} D_{-}^{d^{-}}\right) \bar{u}_{j, k}^{n}\left(y-y_{k}\right)^{2},
\end{aligned}
$$

with $\hat{\theta}_{j, k}^{n}, \hat{s}_{j, k}^{+}$and $\hat{s}_{j, k}^{-}$, given by (B.10), (B.8) and (B.9), respectively.

Acknowledgements. We would like to thank Smadar Karni for valuable suggestions. We would also like to thank Benoit Perthame for fruitful discussions. The work of A. Kurganov was supported in part by the NSF Group Infrastructure Grant and the NSF Grant DMS-0073631. The work of D. Levy was supported in part by the NSF under Career Grant No. DMS-0133511.

\section{REFERENCES}

[1] A. Abdulle, Fourth Order Chebyshev Methods with Recurrence Relation. SIAM J. Sci. Comput. 23 (2002) 2041-2054.

[2] A. Abdulle and A. Medovikov, Second Order Chebyshev Methods Based on Orthogonal Polynomials. Numer. Math. 90 (2001) $1-18$.

[3] P. Arminjon and M.-C. Viallon, Généralisation du schéma de Nessyahu-Tadmor pour une équation hyperbolique à deux dimensions d'espace. C. R. Acad. Sci. Paris Sér. I Math. t. 320 (1995) 85-88.

[4] P. Arminjon, M.-C. Viallon and A. Madrane, A Finite Volume Extension of the Lax-Friedrichs and Nessyahu-Tadmor Schemes for Conservation Laws on Unstructured Grids. Int. J. Comput. Fluid Dyn. 9 (1997) 1-22.

[5] E. Audusse, M.O. Bristeau and B. Perthame, Kinetic Schemes for Saint-Venant Equations With Source Terms on Unstructured Grids. INRIA Report RR-3989 (2000).

[6] A. Bermudez and M.E. Vasquez, Upwind Methods for Hyperbolic Conservation Laws With Source Terms. Comput. E5 Fluids 23 (1994) 1049-1071.

[7] F. Bianco, G. Puppo and G. Russo, High Order Central Schemes for Hyperbolic Systems of Conservation Laws. SIAM J. Sci. Comput. 21 (1999) 294-322.

[8] T. Buffard, T. Gallouët and J.-M. Hérard, A Sequel to a Rough Godunov Scheme. Application to Real Gas Flows. Comput. E Fluids 29-7 (2000) 813-847.

[9] S. Gottlieb, C.-W. Shu and E. Tadmor, High Order Time Discretization Methods with the Strong Stability Property. SIAM Rev. 43 (2001) 89-112.

[10] K.O. Friedrichs and P.D. Lax, Systems of Conservation Equations with a Convex Extension. Proc. Nat. Acad. Sci. USA 68 (1971) 1686-1688.

[11] T. Gallouët, J.-M. Hérard and N. Seguin, Some Approximate Godunov Schemes to Compute Shallow-Water Equations with Topography. Computers and Fluids (to appear).

[12] J.F. Gerbeau and B. Perthame, Derivation of Viscous Saint-Venant System for Laminar Shallow Water; Numerical Validation. Discrete Contin. Dynam. Systems Ser. B 1 (2001) 89-102.

[13] L. Gosse, A Well-Balanced Scheme Using Non-Conservative Products Designed for Hyperbolic Systems of Conservation Laws With Source Terms. Math. Models Methods Appl. Sci. 11 (2001) 339-365.

[14] A. Harten, B. Engquist, S. Osher and S.R. Chakravarthy, Uniformly High Order Accurate Essentially Non-Oscillatory Schemes III. J. Comput. Phys. 71 (1987) 231-303. 
[15] G.-S. Jiang and E. Tadmor, Nonoscillatory Central Schemes for Multidimensional Hyperbolic Conservation Laws. SIAM J. Sci. Comput. 19 (1998) 1892-1917.

[16] S. Jin, A Steady-state Capturing Method for Hyperbolic System with Geometrical Source Terms. ESAIM: M2AN 35 (2001) 631-645.

[17] A. Kurganov and D. Levy, A Third-Order Semi-Discrete Scheme for Conservation Laws and Convection-Diffusion Equations. SIAM J. Sci. Comput. 22 (2000) 1461-1488.

[18] A. Kurganov, S. Noelle and G. Petrova, Semi-Discrete Central-Upwind Schemes for Hyperbolic Conservation Laws and Hamilton-Jacobi Equations. SIAM J. Sci. Comput. 23 (2001) 707-740.

[19] A. Kurganov and G. Petrova, A Third-Order Semi-Discrete Genuinely Multidimensional Central Scheme for Hyperbolic Conservation Laws and Related Problems. Numer. Math. 88 (2001) 683-729.

[20] A. Kurganov and G. Petrova, Central Schemes and Contact Discontinuities. ESAIM: M2AN 34 (2000) 1259-1275.

[21] A. Kurganov and E. Tadmor, New High-Resolution Central Schemes for Nonlinear Conservation Laws and Convection-Diffusion Equations. J. Comput. Phys. 160 (2000) 214-282.

[22] B. van Leer, Towards the Ultimate Conservative Difference Scheme, V. A Second Order Sequel to Godunov's Method. J. Comput. Phys. 32 (1979) 101-136.

[23] R.J. LeVeque, Balancing Source Terms and Flux Gradients in High-Resolution Godunov Methods: The Quasi-Steady WavePropagation Algorithm. J. Comput. Phys. 146 (1998) 346-365.

[24] R.J. LeVeque and D.S. Bale, Wave Propagation Methods for Conservation Laws with Source Terms, Hyperbolic Problems: Theory, Numerics, Applications, Vol. II, Zürich (1998). Birkhäuser, Basel, Internat. Ser. Numer. Math. 130 (1999) 609-618.

[25] D. Levy, G. Puppo and G. Russo, Central WENO Schemes for Hyperbolic Systems of Conservation Laws. ESAIM: M2AN 33 (1999) $547-571$.

[26] D. Levy, G. Puppo and G. Russo, Compact Central WENO Schemes for Multidimensional Conservation Laws. SIAM J. Sci. Comput. 22 (2000) 656-672.

[27] S.F. Liotta, V. Romano and G. Russo, Central Schemes for Systems of Balance Laws, Hyperbolic Problems: Theory, Numerics, Applications, Vol. II, Zürich (1998). Birkhäuser, Basel, Internat. Ser. Numer. Math. 130 (1999) 651-660.

[28] X.-D. Liu and S. Osher, Nonoscillatory High Order Accurate Self Similar Maximum Principle Satisfying Shock Capturing Schemes. I. SIAM J. Numer. Anal. 33 (1996) 760-779.

[29] X.-D. Liu, S. Osher and T. Chan, Weighted Essentially Non-Oscillatory Schemes. J. Comput. Phys. 115 (1994) $200-212$.

[30] X.-D. Liu and E. Tadmor, Third Order Nonoscillatory Central Scheme for Hyperbolic Conservation Laws. Numer. Math. 79 (1998) 397-425.

[31] A. Medovikov, High Order Explicit Methods for Parabolic Equations. BIT 38 (1998) 372-390.

[32] H. Nessyahu and E. Tadmor, Non-Oscillatory Central Differencing for Hyperbolic Conservation Laws. J. Comput. Phys. 87 (1990) 408-463.

[33] S. Noelle, A Comparison of Third and Second Order Accurate Finite Volume Schemes for the Two-Dimensional Compressible Euler Equations, Hyperbolic Problems: Theory, Numerics, Applications, Vol. I, Zürich (1998). Birkhäuser, Basel, Internat. Ser. Numer. Math. 129 (1999) 757-766.

[34] B. Perthame and C. Simeoni, A Kinetic Scheme for the Saint-Venant System with a Source Term. École Normale Supérieure, Report DMA-01-13. Calcolo 38 (2001) 201-301.

[35] G. Russo, Central Schemes for Balance Laws, Proceedings of HYP2000. Magdeburg (to appear).

[36] A.J.C. de Saint-Venant, Théorie du mouvement non-permanent des eaux, avec application aux crues des rivières et à l'introduction des marées dans leur lit. C. R. Acad. Sci. Paris 73 (1871) 147-154.

[37] C.-W. Shu, Total-Variation-Diminishing Time Discretizations. SIAM J. Sci. Comput. 6 (1988) 1073-1084.

[38] C.-W. Shu and S. Osher, Efficient Implementation of Essentially Non-Oscillatory Shock-Capturing Schemes. J. Comput. Phys. 77 (1988) 439-471.

[39] P.K. Sweby, High Resolution Schemes Using Flux Limiters for Hyperbolic Conservation Laws. SIAM J. Numer. Anal. 21 (1984) 995-1011.

[40] E. Tadmor, Convenient Total Variation Diminishing Conditions for Nonlinear Difference Schemes. SIAM J. Numer. Anal. 25 (1988) 1002-1014. 